BULLETIN (New Series) OF THE

AMERICAN MATHEMATICAL SOCIETY

Volume 33, Number 3, July 1996

\title{
CAYLEY-BACHARACH THEOREMS AND CONJECTURES
}

\author{
DAVID EISENBUD, MARK GREEN, AND JOE HARRIS
}

\begin{abstract}
A theorem of Pappus of Alexandria, proved in the fourth century A.D., began a long development in algebraic geometry. In its changing expressions one can see reflected the changing concerns of the field, from synthetic geometry to projective plane curves to Riemann surfaces to the modern development of schemes and duality. We survey this development historically and use it to motivate a brief treatment of a part of duality theory. We then explain one of the modern developments arising from it, a series of conjectures about the linear conditions imposed by a set of points in projective space on the forms that vanish on them. We give a proof of the conjectures in a new special case.
\end{abstract}

\section{CONTENTS}

Introduction $\quad 295$

Part I: The past $\quad 298$

1.1. Pappas, Pascal, and Chasles 298

1.2. Cayley and Bacharach 303

1.3. The twentieth century 311

1.4. Proof of the final Cayley-Bacharach Theorem 316

Part II: The future? $\quad 318$

2.1. Cayley-Bacharach Conjectures 318

2.2. A proof of Conjecture CB10 in case $r \leq 7 \quad 321$

References $\quad 323$

\section{INTRODUCTION}

Suppose that $\Gamma$ is a set of $\gamma$ distinct points in $\mathbb{R}^{n}$ (or $\mathbb{C}^{n}$ ). In fields ranging from applied mathematics (splines and interpolation) to transcendental numbers, and of course also in algebraic geometry, it is interesting to ask about the polynomial functions that vanish on $\Gamma$. If we substitute the coordinates of a point $p$ of $\Gamma$ for the variables, then the condition that a polynomial $f$ vanishes at $p$ becomes a nontrivial linear condition on the coefficients of $f$. Thus the vanishing of $f$ on $\Gamma$ is ensured by $\gamma$ linear conditions on the coefficients of $f$. These $\gamma$ conditions are linearly independent when applied to the space of $\mathbb{R}\left[x_{1}, \ldots, x_{n}\right]$ of all polynomials

Received by the editors March 24, 1995, and, in revised form, November 3, 1995.

1991 Mathematics Subject Classification. Primary 14N05, 14H05, 14-02; Secondary 13-03, $13 \mathrm{H} 10$.

(C)1996 American Mathematical Society 
because, as is easily seen, there is a polynomial vanishing at all but any given point of $\Gamma$.

However, we are usually interested in some finite-dimensional subspace of $\mathbb{R}\left[x_{1}, \ldots, x_{n}\right]$, typically the space of polynomials of degree at most a given number $d$. In this case the $\gamma$ conditions are generally not independent. As a trivial example, consider the three conditions imposed by three collinear points in $\mathbb{R}^{2}$, and take $m=1$. A linear polynomial vanishing on any two of the points vanishes on the line joining them and thus automatically vanishes on the third point. Thus the three points impose only two independent conditions on the polynomials of degree $\leq 1$. In general, if $\lambda$ of the $\gamma$ conditions imposed by $\Gamma$ suffice to imply all of them, and $\lambda$ is the least such number, then we say that $\Gamma$ imposes $\lambda$ independent conditions on polynomials of degree $\leq m$. Since $\lambda \leq \gamma$, and the natural first estimate for $\lambda$ is that it should be "near" $\gamma$, we concentrate on the difference $\gamma-\lambda$, the "failure of $\Gamma$ to impose independent conditions on polynomials of degree $\leq m$ ". There are of course many variants of the question. Perhaps the most basic (and useful) is to take the points in projective space and to ask about homogeneous forms of degree $m$ instead of polynomials of degree at most $m$.

The Cayley-Bacharach Theorem, in its classical form, may be seen as a result about the number of independent conditions imposed on polynomials of given degree by certain sets of points in the plane. The idea is that if the points lie on some algebraic plane curve $X$ of low degree, then the number of conditions imposed by the points can be related to the geometry of $X$. The result has a long and interesting history, starting with a famous result by Pappus of Alexandria, proved in the fourth century A.D. As the methods and substance of algebraic geometry have changed over the years, the result has been successively generalized, improved, and reinterpreted, and this development continues today.

The first part of this paper is purely expository: in it, we shall trace the evolution of the Cayley-Bacharach Theorem. We give altogether nine versions of the result beginning with Pappus's Theorem and continuing with results of Pascal, Chasles, Cayley, and Bacharach. The modern versions of the Cayley-Bacharach Theorem are many; the ideas used have to do with Gorenstein rings and are due to Macaulay, Gorenstein, Serre, and Bass. We shall give a proof of Chasles's version of the theorem using the methods of projective geometry from the middle of the nineteenth century similar to those used by Chasles, a proof of Bacharach's version using the classical algebraic geometry of linear series on curves in the style of the late nineteenth century, and the current proof by commutative algebra. This last connects the geometric statement of the theorem to the Gorenstein property of the polynomial ring; the Cayley-Bacharach Theorem is finally part of duality theory, and Gorenstein rings seem to intervene in all modern treatments of duality in algebraic geometry. (A little warning about our style of history is in order: We have NOT preserved the terminology and "look" of the old works; rather, we try to interpret what the mathematicians of the last century were doing in modern terms. We realize that such "interpretations" are often more accurately described as "fictions". Our excuse is that our purpose is didactic rather than truly historical.)

Our goal in this part of the paper is to show, in this example, how the ideas used in the modern treatment of the Cayley-Bacharach Theorem flow from classical techniques. In particular we introduce some of the basic developments of modern commutative algebra - the Cohen-Macaulay and Gorenstein properties, the Ext 
functor and the like - in the context of a concrete geometric problem, which may serve to motivate and explain them.

One difficulty we have had in writing this report is that while the beginning of our story is accessible with only the most elementary considerations of curves in the plane, the latter part requires much more advanced ideas; in short, the level is uneven. There are really three distinct stages in the proofs that we describe. The first, as we have said, requires almost nothing but the definitions of projective space and polynomials. The second uses the machinery of linear systems on curves, and ultimately the Riemann-Roch theorem. We have given a fairly complete but very quick description of what we need, so that the exposition is almost self-contained. The third part uses the language of commutative algebra and schemes. We have tried to provide elementary definitions and have included proofs of results that are certainly well known to experts and accessible in advanced textbooks rather than referring the reader elsewhere. In particular we have tried to define and motivate the ideas of commutative algebra that are necessary to understand the first properties of Gorenstein rings. The second and third levels are treated independently - the reader can skip either of them without losing logical continuity.

When reviewing the past, it is always tempting to try to predict the future. In the second part of this paper, we propose a possible next step in Cayley-Bacharach theory, in the form of a conjecture. This conjecture is itself a special case of a more general conjecture on the Hilbert functions of points (or, more generally, zero-dimensional schemes) in projective space, which is described in [EGH1] and [EGH2].

To understand what this conjecture says, observe that the earliest forms of the Cayley-Bacharach Theorem may be interpreted as saying that certain sets of points $\Gamma$ impose independent conditions on forms of certain degrees $d$. Later forms compute the number of independent conditions in terms of the number of conditions imposed by some related set of points $\Lambda$. One problem in using these statements is that one may know no more about $\Lambda$ than about $\Gamma$ ! Thus it is interesting to return and ask what sets of points actually impose independent conditions on forms of degree $d$. Our conjectures are related to this question. To understand the form they take, note first that if a set of points $\Gamma$ fails to impose independent conditions on forms of degree $d$, then any larger set of points fails too. Thus it is interesting to ask about the minimal sets of points that fail to impose independent conditions on forms of degree $m$. In the classical Cayley-Bacharach setting the points in question are always given as a subset of the intersection of two curves of given degrees without common components. Our conjectures correspondingly concern subsets of sets of points that are intersections of $n$ hypersurfaces of given degrees $d_{1}, \ldots, d_{n}$ in $\mathbb{P}^{n}$, whose intersection contains no curves. Conjecturally they give the smallest number of points in such an intersection that can fail to impose independent conditions on forms of given degree $m$.

We have succeeded in verifying this conjecture in a number of cases; we conclude the paper by giving the proof in one of these cases.

There is another direction in which current activity is extending the CayleyBacharach Theorem: Davis, Geramita, Robbiano, Kreuzer, and others have defined "Cayley-Bacharach schemes" and studied their properties. For an idea of what is going on, the reader may consult Geramita, Kreuzer, and Robbiano [GKR]. We have excluded this material only because nine versions of the theorem seemed 


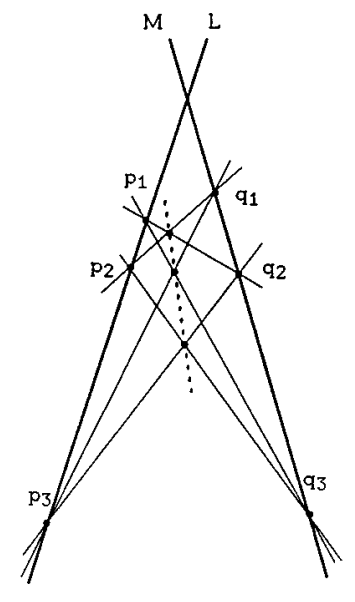

FIGURE 1

enough, and our purpose of introducing some of the commutative algebra associated with Gorenstein rings was already served by what is here.

In what follows we have used the term plane curve always for algebraic plane curve. The field of definition is actually unimportant. Of course it was taken as $\mathbb{R}$ originally, and later as $\mathbb{C}$, and the naive reader would do well to think of these cases, but the expert will have no difficulty in adapting our arguments to the general case.

\section{PART I: THE PAST}

1.1. Pappas, Pascal, and Chasles. The first case of the Cayley-Bacharach Theorem to have been discovered is literally ancient: it appears as Proposition 139 of Book VII of the Mathematical Collection of Pappus of Alexandria in the fourth century A.D. (see Coxeter [Co]).

Theorem CB1 (Pappus's Theorem). Let $L$ and $M$ be two lines in the plane. Let $p_{1}, p_{2}$, and $p_{3}$ be distinct points of $L$, and let $q_{1}, q_{2}$, and $q_{3}$ be distinct points of $M$, all distinct from the point $L \cap M$. If for each $j \neq k \in\{1,2,3\}$ we let $r_{j k}$ be the point of intersection of the lines $\overline{p_{j} q_{k}}$ and $\overline{p_{k} q_{j}}$, then the three points $r_{j k}$ are collinear (see Figure 1).

Pappus's original proof used, in Coxeter's words, "a laborious development of Euclid's methods." It is interesting to note that some notion of the projective plane (not formally introduced until very much later) is necessary if the statement we have given of Pappus's Theorem is to be true in all cases: It is perfectly possible to choose the points so that the lines $\overline{p_{1} q_{2}}$ and $\overline{p_{2} q_{1}}$ are parallel, in which case we would say that the "point" $r_{12}$ is on the line at infinity; the appropriate statement can be made in nonprojective terms by saying that in this case the line through the points $r_{13}$ and $r_{23}$ is parallel to the lines $\overline{p_{1} q_{2}}$ and $\overline{p_{2} q_{1}}$. A still more degenerate case occurs when both the pairs of lines $\overline{p_{1} q_{2}}, \overline{p_{2} q_{1}}$ and $\overline{p_{1} q_{3}}, \overline{p_{3} q_{1}}$ are parallel; then the statement is that all three of the points $r_{j k}$ lie on the line at infinity or, in elementary terms, that the last pair of lines $-\overline{p_{2} q_{3}}, \overline{p_{3} q_{2}}$ - is parallel too. It may be seen from this simple example what a simplification the projective plane introduced! We shall not give a separate proof of Pappus's Theorem, since it is an immediate special case of the following theorem. 


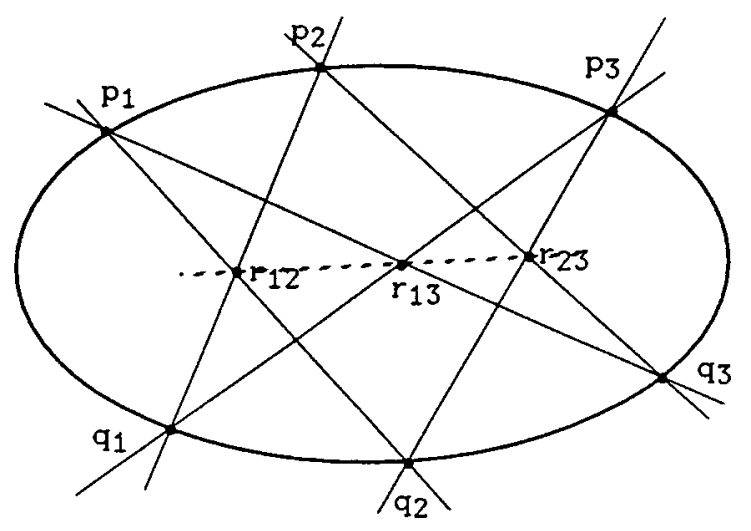

FIGURE 2

In the early part of the seventeenth century there was a new interest in geometry. It included the geometry of perspective, which in the hands of Desargues became the geometry of projective space: Desargues's fundamental work of 1639 formally introduced the points at infinity and the line made from them. There was also a new reason to be interested in the geometry of conics, coming from Kepler's amazing discoveries about the orbits of planets in 1609. The second avatar of the Cayley-Bacharach Theorem that appeared is a famous result of Pascal (again, see Coxeter [Co]) that built on both these developments. The statement was for Pascal a fundamental tool, by means of which he proved many properties of conics. It was published under the title "Essay Pour Les Coniques" in a petit placard en forme d'affiche - that is, a one page handbill - in 1640 and is reproduced in Struik [S]. The proof was not included. Leibniz reports in a letter in 1676 that he studied it when he visited Paris, so we know that Pascal had a proof, but the manuscript has disappeared.

Theorem CB2 (Pascal's Theorem). If a hexagon is inscribed in a conic in the projective plane, then the opposite sides of the hexagon meet in three collinear points.

The line at infinity of the projective plane is again required, as the reader may see by considering the case of a regular hexagon inscribed in a circle. Pascal was immediately inspired by Desargues's work and seems to have been quite aware of this situation. ${ }^{1}$

A little notation will make the relation of Pascal's Theorem to Pappus's Theorem more transparent: Let $C$ be the plane conic, and let $p_{1}, p_{2}, p_{3}$ and $q_{1}, q_{2}$, and $q_{3}$ be six distinct points on $C$. These points determine an inscribed hexagon if we join adjacent points in the order $p_{1}, q_{2}, p_{3}, q_{1}, p_{2}, q_{3}, p_{1}$. This order is chosen so that the "opposite sides" are the pairs of lines $\overline{p_{j} q_{k}}$ and $\overline{p_{k} q_{j}}$ where $i \neq j \in\{1,2,3\}$. If we denote by $r_{j k}$ the point of intersection of these opposite sides, then the assertion is that the three points $r_{j k}$ are collinear, as in Figure 2.

\footnotetext{
${ }^{1}$ Pascal wrote in his Essai: “... M. Desargues of Lyons [is] one of the great minds of this time, and one of the best versed in mathematics, particularly in conics, whose writings on this subject, although few in number, give abundant proof of his knowledge to those who seek for information. I should like to say that I owe the little that I have found on this subject to his writings, and that I have tried to imitate his method, as far as possible, in which he has treated the subject...." (translation from Struik $[\mathrm{S}]$ ).
} 


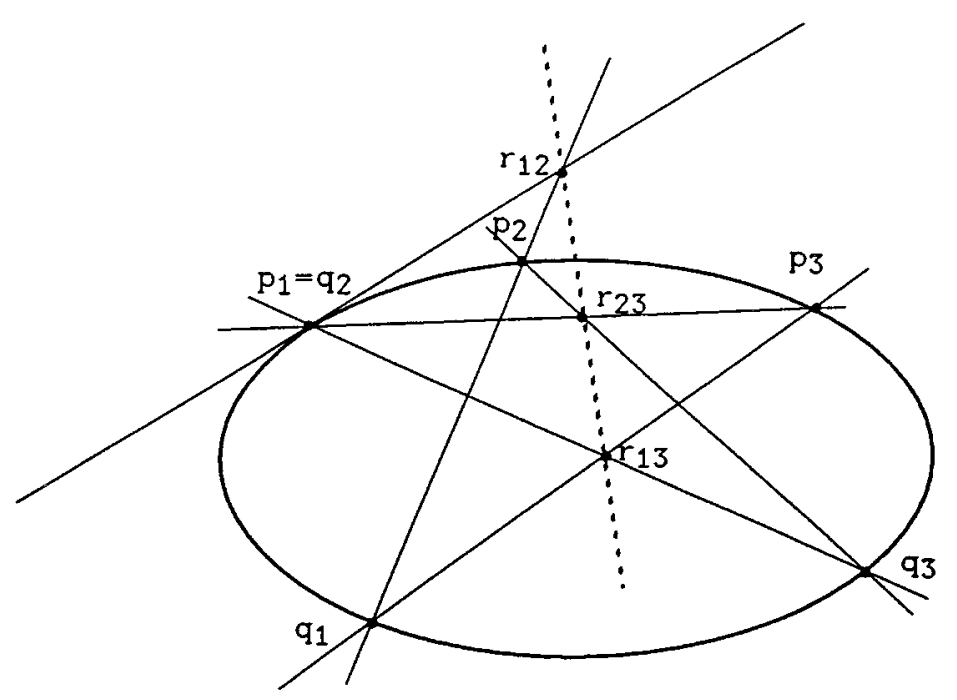

Figure 3. Pascal's construction of the tangent line (at $\left.p_{1}=q_{1}\right)$ to a conic.

Pappus's Theorem becomes a special case of Pascal's Theorem if we allow the word "conic" to refer not only to an irreducible conic such as an ellipse or hyperbola but also to the union of two straight lines $L$ and $M$, which is a kind of limiting position of a hyperbola; the six points $p_{i}$ and $q_{i}$ must be taken with all the $p_{i}$ on one line and all the $q_{i}$ on the other, and all distinct from the intersection of the two lines, or the statement of Pascal's Theorem becomes indeterminate (two of the lines $\overline{p_{j} q_{k}}$ and $\overline{p_{k} q_{j}}$ coincide) or trivial (all the points lie on one of $L$ and $M$ ). From the algebraic point of view, this is natural because then a "conic" is simply the zero locus of any nonzero quadratic polynomial, including products of linear forms. The proof we shall give below applies to all such cases, though we could also deduce Pappus's Theorem from Pascal's by "continuity", an important if slightly imprecise method of the early projective geometers, arguing that the six lines $\overline{p_{j} q_{k}}$ and $\overline{p_{k} q_{j}}$ appearing in Pappus's Theorem are the limiting positions of the sides of a hexagon inscribed in a conic as in Pascal's Theorem.

One of Pascal's many applications of his theorem concerns the "degenerate" case $p_{1}=q_{2}$ : In this case he interprets the line $\overline{p_{1} q_{2}}$ of the "hexagon" to be tangent to the conic at $p_{1}=q_{2}$; see Figure 3 . Since the theorem constructs two further lines that meet at a point of the line $\overline{p_{1} q_{2}}$, namely, $\overline{p_{2} q_{1}}$, and the line through $r_{13}$ and $r_{23}$, it can be used to construct the tangent to the conic at $p_{1}$. This application reflects a modern concern for what happens in the degenerate cases of geometric theorems; the justification for treating the degenerate chord as a tangent line would have been phrased by Pascal in terms of continuity, but would now be justified in the language of schemes. In all the subsequent Cayley-Bacharach theorems that we shall discuss, such degenerate cases are allowed, and the proofs that we shall give in the most general cases explicitly include them.

A third development in the first half of the seventeenth century put the work on the geometry of projective conics into the shade: this was the introduction by Descartes of coordinate geometry. Perhaps because of the excitement generated by 
this development, along with the infinitesimal calculus, geometry moved in other directions, and it was about two hundred years before Pascal's Theorem was fundamentally reexamined and extended.

To understand the nature of the extension, note that in the transition from Pappus's Theorem to Pascal's Theorem the two lines $L$ and $M$ are confounded into a single conic and the two separate groups of points $\left\{p_{1}, p_{2}, p_{3}\right\}$ and $\left\{q_{1}, q_{2}, q_{3}\right\}$ are seen to be an arbitrary subdivision of a single group of six points on the conic, the vertices of the hexagon. In both theorems we construct three further points $r_{i j}$ and a line on which they lie. It was the beautiful insight of Michel Chasles, published in his Traité des Sections Coniques [Ch], that this last line could be combined with the conic in Pascal's Theorem and confounded into a cubic plane curve and that the group of six points and the remaining three could be taken to be any nine points on the cubic, with none distinguished from another. It seems plausible that coordinates for the projective plane, which had recently been defined by Möbius and (in a way closer to the modern one) by Plücker, were necessary before arbitrary plane curves of higher degree, such as cubics, became attractive. In any case, Chasles's result is both simpler and more powerful than Pascal's. It is the result that commonly (though incorrectly) goes under the name Cayley-Bacharach.

Theorem CB3 (Chasles). Let $X_{1}, X_{2} \subset \mathbb{P}^{2}$ be cubic plane curves meeting in nine points $p_{1}, \ldots, p_{9}$. If $X \subset \mathbb{P}^{2}$ is any cubic containing $p_{1}, \ldots, p_{8}$, then $X$ contains $p_{9}$ as well.

Pascal's Theorem follows if we take the cubics $X_{1}$ and $X_{2}$ to be the triangles formed by alternate edges of the hexagon, $X_{1}=\overline{p_{1} q_{2}} \cup \overline{p_{2} q_{3}} \cup \overline{p_{3} q_{1}}$ and $X_{2}=$ $\overline{p_{1} q_{3}} \cup \overline{p_{2} q_{1}} \cup \overline{p_{3} q_{2}}$ in Figure 3, and take $X$ to be the union of $C$ with the line $\overline{r_{12} r_{13}}$. The point $r_{23}$ also lies on $X_{1} \cap X_{2}$, so Chasles's Theorem says it must lie on the union of $C$ and $\overline{r_{12} r_{13}}$. Since it does not lie on $C$, it must lie on $\overline{r_{12} r_{13}}$; that is, $r_{23}$ is collinear with $r_{12}$ and $r_{13}$; as required. The theorem of Pappus is the degenerate case in which $X$ is the union of $L, M$, and $\overline{r_{1} r_{2}}$.

Chasles's Theorem is the last in the sequence of theorems stated here that can be proved by purely elementary means, i.e., without invoking the residue theorem or some other form of duality theory. We shall prove it without making any assumptions about the smoothness or irreducibility of $X_{1}$ and $X_{2}$ - that is, they can be the zero loci of any homogeneous cubic polynomials $F_{1}, F_{2}$ on $\mathbb{P}^{2}$. All we shall need is the classical theorem of Bézout: Plane curves of degrees $d$ and $e$ cannot meet in more than $d \cdot e$ points unless they have a component in common (that is, unless the equations defining them have a common factor). A refined form of Bézout's Theorem says that if the curves have no common component and meet in $d \cdot e$ distinct points, then the curves must each be nonsingular at these points and must meet transversely. In particular the hypotheses of Theorem CB3 rule out the possibility of $X_{1}$ or $X_{2}$ having multiple components, that is, $F_{1}$ or $F_{2}$ having repeated factors. (In fact, the later versions of Cayley-Bacharach will apply to give very interesting and nontrivial statements even in these cases.)

Proof of Chasles's Theorem. We introduce a piece of classical terminology: If $\Gamma=$ $\left\{p_{1}, \ldots, p_{m}\right\} \subset \mathbb{P}^{2}$ is any collection of $m$ distinct points, we shall say that $\Gamma$ imposes $l$ conditions on polynomials of degree $d$ if in the vector space of polynomials of degree $d$ on $\mathbb{P}^{2}$ the subspace of those vanishing at $p_{1}, \ldots, p_{m}$ has codimension $l$, or equivalently if in the projective space of curves of degree $d$ the subspace of those 
containing $\Gamma$ has codimension $l$. We denote the number of conditions imposed by $\Gamma$ on forms of degree $d$ by $h_{\Gamma}(d)$, and call $h_{\Gamma}$ the Hilbert function of $\Gamma$.

With this language, Chasles's Theorem is equivalent to the following statement: If $\Gamma=\left\{p_{1}, \ldots, p_{9}\right\}$ is the intersection of two plane cubics and $\Gamma^{\prime}=\left\{p_{1}, \ldots, p_{8}\right\}$ is any subset omitting one point, then $\Gamma$ and $\Gamma^{\prime}$ impose the same number of conditions on cubics. We shall actually prove the stronger statement that $\Gamma$ and $\Gamma^{\prime}$ each impose exactly eight conditions on cubics; that is, that the eight points of $\Gamma^{\prime}$ impose independent conditions on cubics and the ninth point in $\Gamma$ imposes a condition dependent on these eight.

Part of this is obvious: the nine points of $\Gamma$ visibly fail to impose independent conditions on cubics, since the 10-dimensional vector space of cubic polynomials contains at least a two-dimensional subspace of polynomials vanishing on $\Gamma$, namely, that spanned by the defining equations $F_{1}, F_{2}$ of $X_{1}$ and $X_{2}$. The remaining ingredient of the proof is the "only if" part of the $d=3$ case of the following proposition, which for clarity we shall state and prove for arbitrary $d$.

Proposition 1. Let $\Omega=\left\{p_{1}, \ldots, p_{n}\right\} \subset \mathbb{P}^{2}$ be any collection of $n \leq 2 d+2$ distinct points. The points of $\Omega$ fail to impose independent conditions on curves of degree $d$ if and only if either $d+2$ of the points of $\Omega$ are collinear or $n=2 d+2$ and $\Omega$ is contained in a conic.

This line of thought leads to the numerical character of Gruson-Peskine and to a theorem of Ellia-Peskine [EP] giving a general criterion forcing a subset of a set of points in the projective plane to lie on a curve of low degree.

To complete the proof of Chasles's Theorem using Proposition 1, it suffices by what we have already said to show that if $\Gamma^{\prime}$ consists of eight of the nine points in which two plane cubics intersect, then $\Gamma^{\prime}$ imposes independent conditions on cubics. We take $\Omega=\Gamma^{\prime}$, so $n=8$, and $d=3$ in Proposition 1. By Bézout's Theorem, if $\Gamma^{\prime}$ lay on a conic $C$, then both $X_{1}$ and $X_{2}$ would have to contain (a component of) $C$; and similarly if four or more points of $\Gamma$ lay on a line $L$, then $X_{1}$ and $X_{2}$ would necessarily contain $L$. Since we have supposed that $X_{1}$ and $X_{2}$ have no component in common, this is impossible.

Proof of Proposition 1. The "if" direction of the proposition is easy: If $d+2$ of the points of $\Omega$ lie on a line $L$, then by Bézout's Theorem any curve of degree $d$ containing $\Omega$ must contain $L$. The subset of curves of degree $d$ containing $L$ has the same dimension as the set of curves of degree $d-1$, so the codimension of the set of curves containing $L$ is only $\left(\begin{array}{c}d+2 \\ 2\end{array}\right)-\left(\begin{array}{c}d+1 \\ 2\end{array}\right)=d+1$. The remaining $n-(d+2)$ points of $\Omega$ impose at most $n-(d+2)$ conditions on curves of degree $d$, so we see that $\Omega$ imposes at most $n-1$ conditions. A similar argument, using the fact that it is only $2 d+1$ conditions to contain an irreducible conic, works in the second case.

For the more serious "only if" direction, we do induction first on the degree $d$ and second on the number $n$ of points. By the induction hypothesis on the number $n$ of points we may assume that any proper subset of $\Omega$ does impose independent conditions on curves of degree $d$. Thus the statement that $\Omega$ itself fails to impose independent conditions amounts to saying that any plane curve of degree $d$ containing all but one of the points of $\Omega$ contains $\Omega$.

To start the inductions, we note first that Proposition 1 is trivial for $d=1$ : any set $\Omega$ of $n \leq 4$ points in the plane fails to impose independent conditions on lines if and only if either $n=3=d+2$ and the points of $\Omega$ are all collinear, or $n=4$. 
Second, for arbitrary $d$, the result is easy for $n \leq d+1$ : it suffices by the remark above to exhibit a curve of degree $d$ containing all but one given point $p_{n}$ of $\Omega$, and we may do this by taking the union of general lines $L_{i}$ through $p_{i}$ for $i=1, \ldots, n-1$ and an arbitrary plane curve of degree $d-n+1$ not passing through $p_{n}$.

We now take $d$ arbitrary and suppose that $n>d+1$. Suppose first that $\Omega$ contains $d+1$ points lying on a line $L$. Assume that no further point of $\Omega$ lies on $L$, and let $\Omega^{\prime} \subset \Omega$ be the complementary set of $n-d-1 \leq d+1$ points of $\Omega$. We claim that $\Omega^{\prime}$ must fail to impose independent conditions on curves of degree $d-1$; otherwise, we could find a curve of $X$ degree $d-1$ containing all but any one point of $\Omega^{\prime}$, and then the union $L \cup X$ would be a curve of degree $d$ containing all but one point of $\Omega$. By induction $\Omega^{\prime}$ must consist of exactly $d+1$ points on a line $M$. Thus either $L$ contains $d+2$ points of $\Omega$ or $n=2 d+2$ and $\Omega$ lies on the conic $L \cup M$.

Next suppose only that some line $L$ contains $l \geq 3$ points of $\Omega$. By the same argument as in the last paragraph, the remaining $n-l$ points of $\Omega$ must fail to impose independent conditions on curves of degree $d-1$ and so must include at least $d+1$ collinear points. We are thus back in the case considered in the preceding paragraph.

We are now done except in the case where $\Omega$ contains no three collinear points. Choose any three points $p_{1}, p_{2}, p_{3} \in \Omega$, and let $\Omega^{\prime}$ be the complement of these three. If for any $i$ the points of $\Omega^{\prime} \cup\left\{p_{i}\right\}$ impose independent conditions on curves of degree $d-1$, we are done: for then we can find a curve $C$ of degree $d-1$ containing $\Omega^{\prime}$ but not $p_{i}$, and the union of this curve and the line joining $p_{j}$ and $p_{k}$ is a curve of degree $d$ containing all but exactly one point of $\Omega$. Thus we may assume that $\Omega^{\prime} \cup\left\{p_{i}\right\}$ fails to impose independent conditions on curves of degree $d-1$. Since it cannot contain $d+1$ collinear points, we have by induction $n=2 d+2$, and for each $i$ the set $\Omega^{\prime} \cup\left\{p_{i}\right\}$ lies on a conic $C_{i}$. Note that in case $d=2$ we are done, since six points fail to impose independent conditions on conics if and only if they lie on a conic. On the other hand, if $d \geq 3$, then $\Omega^{\prime}$ contains at least five points, no three collinear, and so there can be at most one conic containing $\Omega$. Thus all the conics $C_{i}$ must be equal to a single conic curve $C$, which then contains all of $\Omega$.

There is a much quicker proof of Theorem CB3, which exploits the relation of the theorem to residue theory; since this relation belongs to the next part of our story, we shall give it there.

1.2. Cayley and Bacharach. Arthur Cayley, probably the most distinguished mathematician in this story, does not play a glorious role in it. Chasles's book appeared when he was sixteen. In 1843, when he was twenty-two, he published a note stating an extension of Chasles's result to the case of intersections of curves of degree higher than 3. The basis of this extension was again the idea of counting conditions imposed by sets of points. His first observation was that if curves $X_{1}$ and $X_{2}$ of degrees $d$ and $e$ meet in a collection $\Gamma$ of $d \cdot e$ points, then for any $k$ the number $h_{\Gamma}(k)$ of conditions imposed by $\Gamma$ on forms of degree $k$ is independent of the choice of curves $X_{1}$ and $X_{2}$; it can be written down explicitly as

$$
h_{\Gamma}(k)=\left(\begin{array}{c}
k+2 \\
2
\end{array}\right)-\left(\begin{array}{c}
k-d+2 \\
2
\end{array}\right)-\left(\begin{array}{c}
k-e+2 \\
2
\end{array}\right)+\left(\begin{array}{c}
k-d-e+2 \\
2
\end{array}\right)
$$

where the binomial coefficient $\left(\begin{array}{l}a \\ 2\end{array}\right)$ is to be interpreted as 0 if $a<2$. He went on to conclude that if $\Gamma^{\prime}$ is any subset of $h_{\Gamma}(k)$ points of $\Gamma$, then a form of degree $k$ 
vanishing on the points of $\Gamma^{\prime}$ must vanish on $\Gamma$. (The case $d=e=k=3$ is Chasles' Theorem.)

Both parts of Cayley's statement are rather remarkable. The first part is equivalent to a special case of the whole: any curve $X$ passing through all the points of the intersection of $X_{1}$ and $X_{2}$ is defined by a polynomial that is a linear combination (with polynomial coefficients) of the polynomials defining $X_{1}$ and $X_{2}$. That is, if $F=0, G=0$, and $H=0$ are the equations of $X_{1}, X_{2}$, and $X$ respectively, then there exist polynomials $A$ and $B$ such that $H=A F+B G$. This assertion was used by Cayley in the case where the two curves meet transversely. He apparently regarded it as evident; he applies it without comment or reference. But the statement is rather subtle: in modern language, it says that the polynomial ring in three variables is Cohen-Macaulay. It was finally given a proof by Max Noether, first in the special case Cayley used [Ca, p. 314] and then in general [No] in a paper devoted to filling this gap", under the name of the "Fundamental Theorem of Algebraic Functions" (now often - and perhaps unfortunately - called "Max Noether's $A F+B G$ Theorem").

Given Noether's Theorem and the fact that the dimension of the space of all forms of degree $k$ in three variables is $\left(\begin{array}{c}k+2 \\ 2\end{array}\right)$, the formula for $h_{\Gamma}$ given above is easy: it just says that the vector space of forms of degree $k$ that can be written as $A F+B G$ is the sum of the dimension of the space of possible $A$ 's (of degree $k-d$ ) and the space of possible $B$ 's (of degree $k-e$ ), minus the dimension of the space of pairs $(A, B)$ such that $A F+B G=0$. Since $F$ and $G$ have no factors in common, $A F+B G=0$ can hold only if $A$ is a multiple of $G$, and $B$ is minus the corresponding multiple of $F$, and the multiplier has degree $k-d-e$. Thus the dimension of the space of these pairs is the dimension of the space of forms of degree $k-d-e$.

By the argument given in the proof of Chasles's Theorem above, the second part of Cayley's statement amounts to a broad generalization of Proposition 1but without any hypotheses! What is necessary is precisely the assertion that the points of the subset $\Gamma^{\prime}$ impose independent conditions on forms of degree $k$. This would of course be true if the points were general points. Cayley does comment on this point. He says, "... and though the [...] points are not perfectly arbitrary, there appears to be no reason why the relation between the positions of these points should be such as to prevent" the conditions from being independent.

Despite this "appearance", points may have their own reasons and can indeed fail to impose independent conditions. The conclusion of Cayley's Theorem is simply false! Perhaps the first example is the following: Let $L$ be a line in the plane, and let $\Gamma^{\prime}=\left\{p_{1}, p_{2}, p_{3}\right\}$ be three points on $L$. Let $X_{1}$ and $X_{2}$ be two nonsingular cubic curves containing $\Gamma^{\prime}$. It is easy to arrange that $X_{1}$ and $X_{2}$ meet in a collection of nine distinct points $\Gamma$. We have $h_{\Gamma}(1)=3$ (that is, $\Gamma$ does not lie on any lines), and thus if the points of $\Gamma^{\prime}$ imposed independent conditions on lines, every line containing them would contain all of $\Gamma$ (that is, there would be no lines containing them). This is of course nonsense: The points of $\Gamma^{\prime}$ do lie on the line $L$, and none of the other points of $X_{i}$ lie on $L$, or $L$ would be a component of $X_{i}$.

\footnotetext{
${ }^{2}$ Noether writes, "In einer Reihe von geometrischen und functionentheoretischen Arbeiten findet sich eine Lücke, die das Folgende auszufüllen bestimmt ist." ("In a series of geometric and function-theoretic papers there is a gap, which the following is intended to fill.") It would be interesting to know just when and how, in between 1843 and 1873, it came to be considered a gap.
} 
What is needed is a way of measuring the dependence of the conditions imposed by the points of $\Gamma^{\prime}$. More powerful tools were soon to be available. Riemann's remaking of complex function theory led Brill and Noether to an extensive theory of linear series on algebraic curves; Noether's Fundamental Theorem of Algebraic Functions was in fact one of the tools they developed for the purpose. This theory was powerful enough to supply the missing term in Cayley's equation; the fundamental paper of Brill and Noether appeared in 1874, and its tools were used by Bacharach to correct Cayley's error. Bacharach's work seems to have earned him a professorship in Erlangen. He presented his findings in 1881 in his inaugural dissertation. Perhaps the problem with Cayley's "Theorem" had been recognized for some time. Bacharach published a somewhat extended version of his results in [Ba]: this paper contains the subtle missing term, and we shall give his statement below. In the case just given it amounts to the observation that the fact that the points of $\Gamma^{\prime}$ impose dependent conditions on lines is equivalent to the statement that the "residual" set $\Gamma^{\prime \prime}:=\Gamma-\Gamma^{\prime}$ imposes dependent conditions on conics; that is, the other six points of $\Gamma$ actually lie on a conic.

It may be illuminating to see the ideas of linear series applied first in the simple case studied by Chasles; in fact, without any analysis or struggle, the method shows that if two curves of any degree $d$ intersect a curve of degree $e \geq 3$ in sets $\Gamma$ and $\Gamma^{\prime}$ differing by at most one point, then in fact $\Gamma=\Gamma^{\prime}$. We shall give the easy proof after developing the ideas from the theory of curves on which it is based.

For simplicity, the reader may assume at this point that the ground field is the complex numbers, although the expert will have no trouble adapting the argument to the general case. If $X \subset \mathbb{P}^{2}$ is a nonsingular algebraic plane curve of degree $d$, then by a divisor $D$ on $X$ we mean a formal linear combination, with integer coefficients, of the points of $X$; by the degree of $D$ we will mean the sum of the coefficients. The divisor is called effective if all the coefficients are nonnegative. Any rational function $f$ on $X$ determines a divisor (f), the divisor of its zeros minus the divisor of its poles. Divisors $D$ and $D^{\prime}$ are said to be linearly equivalent, written $D \sim D^{\prime}$, if they differ by the divisor of a rational function.

The same notions can be defined for an arbitrary plane curve $X$ (and even for more general curves), but some care is necessary. First, if $X$ is irreducible and singular and the divisors in question involve only smooth points, the most interesting situation for us, we need only change the definition of linear equivalence to require the rational function $f$ to be regular and nonzero at the singular points of the curve, so that the linear equivalence takes place entirely inside the smooth locus. If $X$ is reducible, then in addition we must require that the rational functions have only isolated zeros and poles on the curve, not along a whole component. If we wish to include the singular points as divisors in some way, there is an additional complication, one that was not faced squarely in the nineteenth century: divisors must be interpreted as what are now called Cartier divisors. For this final refinement see any modern book on algebraic geometry, for example Hartshorne $[\mathrm{H}]$.

For example, if $C$ is any plane curve intersecting $X$ only in isolated smooth points of $X$, we define the divisor cut on $X$ by $C$ to be the divisor $C \cdot X:=\Sigma a_{i} p_{i}$, where the $p_{i}$ are the points of intersection of $C$ with $X$ and $a_{i}$ is the multiplicity of intersection of $C$ with $X$ at $p_{i}$. If $C$ and $C^{\prime}$ are plane curves of the same degree not containing $X$, defined by homogeneous polynomials $F$ and $F^{\prime}$, the divisors $C \cdot X$ and $C^{\prime} \cdot X$ are linearly equivalent: their difference is the divisor of the rational 
function $F / F^{\prime}$ restricted to $X$. In this context we may give a refined version of the Bézout Theorem:

Theorem 2 (Bézout). If $C$ is a plane curve of degree e not containing $X$, the degree of the divisor cut on $X$ by $C$ is $d \cdot e$.

One of the central results proved by Brill and Noether was the following. The original version takes the curve $X$ to be irreducible, but the difference is mostly a matter of how the definitions are formulated.

Theorem 3 (Restsatz: Brill-Noether [BN]). If $X$ is a plane curve, then the linear series cut on $X$ by plane curves of any degree $d$ is complete: that is, given a plane curve $C$ of degree $d$ not containing any component of $X$ and a divisor $D$ linearly equivalent to $C \cdot X$, there is a plane curve $C^{\prime}$ of degree $d$ not containing any component of $X$ such that $C^{\prime} \cdot X=D$.

Proof. We need the following form of Max Noether's $A F+B G$ Theorem (which itself is a consequence of Lasker's Unmixedness Theorem; see Theorem 8 below): If a curve $Y$ contains $C \cdot X$, then the equation $H$ of $Y$ may be written as $A F+B G$, where $F$ is the equation of $C$ and $G$ is the equation of $X$. It follows that the curve $Y^{\prime}$ with equation $H-B G$ meets $X$ in the same way $Y$ does; that is, $Y^{\prime} \cdot X=Y \cdot X$, and $Y^{\prime}$ contains $C$ as a component.

The hypothesis of Corollary 5 asserts that there is a rational function $\varphi$ on $X$ whose divisor $(\varphi)$ satisfies $C \cdot X-\Gamma=D+(\varphi)$. Adding $\Gamma$ to both sides, we reduce at once to the case where $\Gamma=0$. The rational function $\varphi$ may be expressed as $P / Q$ where $P$ and $Q$ are homogeneous polynomials in three variables of the same degree. Let $Y$ and $Z$ be the curves defined in the plane by $P$ and $Q$ respectively. We have $C \cdot X+Z \cdot X=D+Y \cdot X$. Multiplying together the equations of $C$ and $Z$, we get the equation of a curve $Z^{\prime}$ such that $Z^{\prime} \cdot X=D+Y \cdot X$. In particular, $Z^{\prime}$ contains $Y \cdot X$. By Max Noether's Theorem, we may replace $Z^{\prime}$ by a curve $Z^{\prime \prime}$ containing $Y$ and such that $Z^{\prime \prime} \cdot X=Z^{\prime} \cdot X=D+Y \cdot X$. If $S$ is the equation of $Z^{\prime \prime}$, then since $Z^{\prime \prime}$ contains $Y$ we see that $P$ divides $S$. If we write $C^{\prime}$ for the curve defined by $S / P$, which we may represent as $C^{\prime}=Z^{\prime \prime}-Y$, then $C^{\prime} \cdot X=D$.

Noether's Fundamental Theorem of Algebraic Functions mentioned above was a fundamental tool in the proof of Theorem 3 given by Brill and Noether. From a modern point of view, this is not surprising. Theorem 3 is the statement that the homogeneous coordinate ring of the curve $X$ has the Cohen-Macaulay property, which is equivalent to saying that the polynomial ring itself has this property, and Noether's Fundamental Theorem is essentially equivalent to this assertion too! See the discussion after Theorem 8 for more information.

Given this, the proof of Chasles's Theorem CB3, and even its generalization given above, becomes trivial: If $X$ is a plane curve of degree $e \geq 3$ and $C$ and $C^{\prime}$ are plane curves of some degree $d$ meeting $X$ in divisors $D$ and $D^{\prime}$ that differ by at most one smooth point, say, $D-D^{\prime}=p-q$, then $p$ and $q$ are linearly equivalent. Choose a general line $L$ through $p$; we will show that $L$ passes through $q$, and thus $p=q$. By the Restsatz there is some line $L^{\prime}$ that cuts out the divisor $L \cdot X-p+q$. Because $e \geq 3$, the divisor $L \cdot X-p$ already contains at least two points, and thus spans $L$, so $L=L^{\prime}$ as required. (Chasles's Theorem is the special case $d=e=3$.)

In fact a much stronger statement, still short of the full version of Bacharach, can be derived by related methods and will play a role in the sequel, so we pause to examine it. 
Theorem CB4. Let $X_{1}, X_{2} \subset \mathbb{P}^{2}$ be plane curves of degrees $d$ and e respectively, meeting in a collection of $d \cdot e$ distinct points $\Gamma=\left\{p_{1}, \ldots, p_{\text {de }}\right\}$. If $C \subset \mathbb{P}^{2}$ is any plane curve of degree $d+e-3$ containing all but one point of $\Gamma$, then $C$ contains all of $\Gamma$.

In the terms of Bacharach's Theorem CB5, this result is true because any set $\Gamma^{\prime}$ consisting of all but one point of $\Gamma$ really does impose independent conditions on forms of degree $d+e-3$.

To apply the Brill-Noether theory in a naive form to this result, we shall have to assume that one of the curves $X_{i}$, say, $X_{1}$, is nonsingular. (The expert could avoid this hypothesis, either by using a more subtle version of Riemann-Roch or by using Bertini's Theorem: Since we are assuming that $\Gamma$ consists of $d \cdot e$ distinct points, the refined form of Bézout's Theorem shows that $X_{1}$ and $X_{2}$ meet transversely, and then a classic theorem of Bertini says that (if $d>e$ ) we can find a nonsingular curve $X_{1}^{\prime}$ that meets $X_{2}$ in the same set of points $\Gamma$ by adding to the equation of $X_{1}$ an appropriate multiple of the polynomial defining $X_{2}$. But since later versions of the theorem will be more general anyway, we shall not go further into this here.) This is in fact not the approach we shall adopt ultimately to prove the final and most general version of the Cayley-Bacharach theorem, and the reader who wishes to can certainly skip the proofs of statements CB4-CB7 or just glance at them to get a sense of the ingredients without suffering any logical gaps.

Using the language of the theory of curves and denoting by $H$ the divisor cut on a plane curve $X$ by a line $L \subset \mathbb{P}^{2}$, we can state a surprising property of the divisor $(d-3) H$, from which Theorem CB4 will follow immediately.

Proposition 4. Let $X \subset \mathbb{P}^{2}$ be a nonsingular plane curve of degree $d$, and let $p$ be a point of $X$. Every effective divisor linearly equivalent to $(d-3) H+p$ actually contains $p$.

This baffling statement loses (some of) its mystery when viewed as a very special case of the Riemann-Roch Theorem (in fact, of Riemann's Theorem itself, though we shall not worry about this distinction). To explain this, we first note that any two rational differential forms on $X$ have a ratio that is a rational function on $X$, and thus the divisor of zeros minus the divisor of poles of any rational differential form is a well-defined divisor class on $X$, called the canonical divisor class, and denoted $K_{X}$. Abusing notation in a traditional way, we shall also denote by $K_{X}$ any divisor in this class. Next, we recall that if the ground field is $\mathbb{C}$, then $X$ may be viewed as a Riemann surface, and as such it has a genus $g$ (the "number of handles"); this invariant and the fact that it is a compact orientable surface specifies its topology completely. There is a miraculous connection between the topology of $X$ and the geometry on $X$ : the degree of $K_{X}$ is $2 g-2$. (In the case of a general ground field, this can be taken as the definition of the genus.) Now for a plane curve it is not hard to compute $K_{X}$ by writing down a specific rational differential form. The result is a special case of what algebraic geometers call the adjunction formula, and it tells us that

$$
K_{X} \sim(d-3) \cdot H .
$$

The divisor $(d-3) H$ that appears in Proposition 4 is "really" $K_{X}$. It is worth noting another consequence of the adjunction formula in passing. By Bézout's Theorem the degree of the divisor $(d-3) H$ is $d(d-3)$, so we get $2 g-2=(d-3) d$, or $g=(d-1)(d-2) / 2$. 
To exploit the identification of $(d-3) H$ with $K_{X}$, we must use the RiemannRoch Theorem, which we now introduce. For any divisor $D$ on $X$, we shall denote by $L(D)$ the vector space of rational functions $f$ such that $D$ plus the divisor of $f$ is effective. We denote the vector space dimension of $L(D)$ by $l(D)$. For example, Liouville's Theorem asserts that the only rational functions with no zeros or poles are the constant functions, so $l(0)=1$.

With these definitions, the Riemann-Roch Theorem asserts that for any divisor $D$ of degree $n$ on $X$,

$$
l(D)=n-g+1+l\left(K_{X}-D\right)
$$

where $g=(d-1)(d-2) / 2$ is the genus of $X$. For example, $l\left(K_{X}\right)=(2 g-2)-g+$ $1+l(0)=g-1+1=g$.

Proof of Proposition 4. Let $p$ be a point of $X$. The degree of the divisor $K_{X}+p$ is $2 g-1$, so by Riemann-Roch we have

$$
l\left(K_{X}+p\right)=(2 g-1)-g+1+l(-p)=g+l(-p) .
$$

But the degree of $-p$ is -1 , so no effective divisor can be equivalent to $-p$; thus $l(-p)=0$. It follows that $l\left(K_{X}+p\right)=g=l\left(K_{X}\right)$. But given any effective divisor $D$ linearly equivalent to $K_{X}$, we may make a divisor $D+p$ linearly equivalent to $K_{X}+p$, the map $D \mapsto D+p$, as a map $L(D) \rightarrow L(D+p)$, is actually a vector space homomorphism. It is obviously injective, and since the two spaces have the same dimension, they must be equal.

Proof of Theorem CB4 (under the assumption that $X_{1}$ is nonsingular). Suppose $C \subset \mathbb{P}^{2}$ is a plane curve of degree $d+e-3$ containing all of $X_{1} \cap X_{2}$ except for the point $p=p_{d e}$. We can write the divisor cut on $X_{1}$ by $C$ as

$$
C \cdot X_{1}=p_{1}+\cdots+p_{d e-1}+q_{1}+\cdots+q_{d(d-3)+1} \cdot
$$

We have $K_{X_{1}} \sim(d-3) \cdot H$, while $p_{1}+\cdots+p_{d e} \sim e \cdot H$ and $C \cdot X_{1} \sim(d+e-3) \cdot H$. Thus we can rewrite the equation as

$$
(d+e-3) \cdot H \sim e \cdot H-p+q_{1}+\cdots+q_{d(d-3)+1}
$$

or, equivalently,

$$
\begin{aligned}
q_{1}+\cdots+q_{d(d-3)+1} & \sim(d-3) \cdot H+p \\
& \sim K_{X_{1}}+p .
\end{aligned}
$$

By Proposition 4 , we get $p \in\left\{q_{1}, \ldots, q_{d(d-3)+1}\right\}$. In particular, $p \in C$.

Another way to express this last argument, more explicitly invoking the Residue Theorem, is to choose $(x, y)$ affine coordinates on the plane; let $f(x, y), g(x, y)$, and $h(x, y)$ be the equations of the curves $X_{1}, X_{2}$, and $C$ in these coordinates; and consider the differentials

$$
\omega=\frac{d x}{\partial f / \partial y}
$$

and

$$
\varphi=\frac{h(x, y)}{g(x, y)} \omega
$$

on $X_{1}$. The form $\omega$ itself is regular and nonzero on the affine part of $X_{1}$, having zeros of order $d-3$ along the divisor cut on $X_{1}$ by the line at $\infty$. It follows that $\varphi$ is regular at the points at $\infty$, with simple poles exactly at the points of $X_{1}$ contained 
in $X_{2}$ but not in $C$. There is at most one such point, namely, $p$. By the Residue Theorem, the sum of the residues of any differential form must be 0 , so a differential on a curve cannot have exactly one simple pole. We conclude that $p \in C$.

A more careful application of Riemann-Roch yields the version of CayleyBacharach that was actually proved in Bacharach [Ba]. It relates the number of conditions imposed on curves of various degrees by two complementary subsets of the intersection of two plane curves. We need one more fact from the general theory of plane curves: Let $X$ be a nonsingular plane curve of degree $d$, and let $C$ be a plane curve of degree $k$. The refined form of Bézout's Theorem says that by counting each point with an appropriate multiplicity, we may regard the intersection of $C$ and $X$ as a divisor on $X$ of degree $d \cdot k$, which we will denote by $C \cdot X$. The fact we want is a corollary of Theorem 3 . We may express it in classical language as

Corollary 5. Let $X$ be a nonsingular plane curve, and let $C$ be any plane curve not containing any component of $X$. Let $\Gamma$ be any effective divisor on $X$. The family of plane curves containing $\Gamma$ cuts out on $X$ the complete linear series of divisors linearly equivalent to $C \cdot X-\Gamma$.

We shall exploit Corollary 5 to express the number of conditions imposed on forms of degree $m$ by a set of points of $X$ in terms that are accessible to the Riemann-Roch Theorem. The precise result is:

Corollary 6. Let $X$ be a smooth plane curve of degree $d$, and let $\Lambda \subset X$ be a set of $\lambda$ points, regarded as a divisor on $X$. The number of linear conditions imposed by $\Lambda$ on forms of degree $m$ is equal to $l(m H)-l(m H-\Lambda)$. In particular, the "failure of $\Lambda$ to impose independent conditions on forms of degree $m$ " is $\lambda-(l(m H)-l(m H-\Lambda))$.

Proof. The "number of linear conditions imposed by $\Lambda$ on forms of degree $m$ " is the dimension $t$ of the vector space of forms of degree $m$ modulo those vanishing on $\Lambda$. The number $l(m H)$ is the dimension of the vector space $L(m H)$, which by Corollary 5 (applied in the case when $\Gamma$ is the empty set) is the dimension of the space of forms of degree $m$ modulo those vanishing on $X$. Similarly, the number $l(m H-\Lambda)$ is by Corollary 5 the dimension of the space of forms of degree $m$ vanishing on $\Lambda$ modulo those vanishing on all of $X$. Thus $t=l(m H)-l(m H-\Lambda)$. The "failure of $\Lambda$ to impose independent conditions is simply the number of points $\lambda$ of $\Lambda$ (the maximal number of conditions that $\Lambda$ could impose) minus the number of conditions actually imposed, or $\lambda-(l(m H)-l(m H-\Lambda))$.

Theorem CB5 (Bacharach). Let $X_{1}, X_{2} \subset \mathbb{P}^{2}$ be plane curves of degrees $d$ and $e$ respectively, intersecting in $d \cdot e$ points $\Gamma=X_{1} \cap X_{2}=\left\{p_{1}, \ldots, p_{d e}\right\}$, and suppose that $\Gamma$ is the disjoint union of subsets $\Gamma^{\prime}$ and $\Gamma^{\prime \prime}$. Set $s=d+e-3$. If $k \leq s$ is a nonnegative integer, then the dimension of the vector space of forms of degree $k$ vanishing on $\Gamma^{\prime}$ (modulo those containing all of $\Gamma$ ) is equal to the failure of $\Gamma^{\prime \prime}$ to impose independent conditions on forms of degree $s-k$.

This theorem is one possible departure point for a beautiful series of mathematical developments known as liaison theory. Liaison is the equivalence relation gotten by allowing a variety $X^{\prime}$ to be replaced by the variety $X^{\prime \prime}$ residual to it for some complete intersection $X$ containing $X^{\prime}$.

Note that Theorem CB4 is just the case where $k=s$ and $\Gamma^{\prime \prime}$ is a single point $p=p_{d e}$ : Since any point $p \in \Gamma$ imposes one independent condition on polynomials of degree 0 , the conclusion is that there are no hypersurfaces of degree $k$ containing 
$\Gamma-\{p\}$ except those containing $\Gamma$. Theorem CB5 says further that any curve of degree $s-1=d+e-4$ containing all but two points of $\Gamma$ contains $\Gamma$ and there exists a curve of degree $s-1$ containing all but three points $p, q, r \in \Gamma$ but not containing $\Gamma$ if and only if $p, q$, and $r$ are collinear, and so on.

Once again we give the proof under the assumption that $X_{1}$ is nonsingular; the more general case may be treated using Bertini's Theorem, as indicated before.

Proof (under the assumption that $X_{1}$ is nonsingular). As before, denote by $H$ the hyperplane divisor on $X_{1}$; we shall consider $\Gamma, \Gamma^{\prime}$, and $\Gamma^{\prime \prime}$ as divisors on $X_{1}$ as well. The dimension of the family of curves of degree $k$ containing $\Gamma^{\prime}$, modulo those containing all of $\Gamma$, is the same as the number of conditions imposed by $\Gamma$ on curves of degree $k$ minus the number of conditions imposed by $\Gamma^{\prime}$, that is, $h_{\Gamma}(k)-h_{\Gamma^{\prime}}(k)$. By Corollary 5 , the families of plane curves of degree $k$ containing $\Gamma$ and containing $\Gamma^{\prime}$ cut on $X_{1}$ the linear series of divisors equivalent to $k \cdot H-\Gamma^{\prime}$ and $k \cdot H-\Gamma=(k-e) \cdot H$ respectively. By Riemann-Roch we have

$$
\begin{aligned}
l(k \cdot H & \left.-\Gamma^{\prime}\right)-l((k-e) \cdot H) \\
= & k d-\gamma^{\prime}-g+l\left((d-3-k) \cdot H+\Gamma^{\prime}\right) \\
& \quad-[(k-e) d-g+l((d-3-k+e) \cdot H)] \\
= & d \cdot e-\gamma^{\prime}-\left[l\left((d-3-k) \cdot H+\Gamma^{\prime}\right)-l((d-3-k+e) \cdot H)\right] .
\end{aligned}
$$

Now, since the divisors $\Gamma^{\prime}$ and $\Gamma^{\prime \prime}$ add up to $e \cdot D$ and their degrees correspondingly add up to $e \cdot d$, we can rewrite this as

$$
=\gamma-\left[l((s-k) \cdot H)-l\left((s-k) H-\Gamma^{\prime \prime}\right)\right]
$$

which is the failure of $\Gamma^{\prime \prime}$ to impose independent conditions on curves of degree $s-k$.

There is an immediate generalization of Theorem CB5 to a statement about the transverse intersection of $n$ hypersurfaces $X_{i}$ of degrees $d_{i}$ in $\mathbb{P}^{n}$. Here the role of the number $d+e-3$ in the preceding statement is played by $s=\Sigma d_{i}-n-1$.

Theorem CB6. Let $X_{1}, \ldots, X_{n}$ be hypersurfaces in $\mathbb{P}^{n}$ of degrees $d_{1}, \ldots, d_{n}$ respectively, meeting transversely, and suppose that the intersection $\Gamma=X_{1} \cap \cdots \cap X_{n}$ is the disjoint union of subsets $\Gamma^{\prime}$ and $\Gamma^{\prime \prime}$. Set $s=\Sigma d_{i}-n-1$. If $k \leq s$ is a nonnegative integer, then the dimension of the family of curves of degree $k$ containing $\Gamma^{\prime}$ (modulo those containing all of $\Gamma$ ) is equal to the failure of $\Gamma^{\prime \prime}$ to impose independent conditions of curves of "complementary" degree $s-k$.

Proof (in case $X=X_{1} \cap \cdots \cap X_{n-1}$ is nonsingular). The proof follows exactly the lines of the proof of the last version, modulo the following changes: in the setting of the current theorem we have $\Gamma \sim d_{n} \cdot H$, so we replace $e$ in the previous proof with $d_{n}$. In the new setting the adjunction formula tells us that

$$
\begin{aligned}
K_{X} & \sim\left(\sum_{1}^{n-1} d_{i}-n-1\right) \cdot H \\
& =\left(s-d_{n}\right) \cdot H,
\end{aligned}
$$

so we replace $d-3$ in the previous proof by $s-d_{n}$. 
1.3. The twentieth century. The next stage in the evolution of the CayleyBacharach Theorem may seem in some sense a purely technical advance: we shall eliminate the hypothesis that the hypersurfaces $X_{i}$ intersect transversely and replace it with the weaker one that they intersect in isolated points; that is, in modern language, we no longer assume that the scheme $\Gamma=X_{1} \cap \cdots \cap X_{n}$ of intersection of the $X_{i}$ is reduced, only that it is zero-dimensional. In fact, though, this is the generalization that in our view transforms the problem and ultimately unifies the approaches above.

Unfortunately, the geometric content of the new version is slightly less intuitive; the intersections must be treated as schemes. Some would even claim that what we are about to do amounts to nothing other than forgetting geometry and working directly in algebra. But we feel that the geometric language still carries the clearest picture. So to read further the reader will have to accept the notion that every ring (or at least every finitely generated algebra over our still unnamed ground field) corresponds to some geometric object, a scheme, in a way extending the correspondence of reduced finitely generated algebras over an algebraically closed field with algebraic sets. For a relatively simple introduction to this notion, the reader may consult Eisenbud-Harris [EH2]. In any case, we shall not use any deep property of this notion, and the algebra that we need we shall introduce from scratch.

Before we can state this version of the Cayley-Bacharach Theorem, we have to generalize the notion of expressing the intersection $X_{1} \cap \cdots \cap X_{n}=\Gamma$ as a disjoint union $\Gamma^{\prime} \cup \Gamma^{\prime \prime}$ to the case where $\Gamma$ is an arbitrary zero-dimensional scheme. In general, we would like to define a notion of "residual subscheme" $\Gamma^{\prime \prime}$ to a subscheme $\Gamma^{\prime}$ of a zero-dimensional scheme $\Gamma$. This should play the role of a "complementary subset", except that the support of $\Gamma^{\prime \prime}$ may overlap with that of $\Gamma^{\prime}$. We want this definition to have two basic properties: the degrees of the two subschemes $\Gamma^{\prime}$ and $\Gamma^{\prime \prime}$ should add up to that of $\Gamma$, and the process should be symmetric; i.e., $\Gamma^{\prime}$ should be in turn the subscheme of $\Gamma$ residual to $\Gamma^{\prime \prime}$. An obvious desideratum for the residual subscheme is that the product of a function vanishing on $\Gamma^{\prime}$ and one vanishing on $\Gamma^{\prime \prime}$ should vanish on $\Gamma$. We shall define the residual subscheme as the maximal subscheme with this property. In other terms:

Definition. Let $\Gamma$ be a zero-dimensional scheme with coordinate ring $A(\Gamma)$. Let $\Gamma^{\prime} \subset \Gamma$ be a closed subscheme and $I_{\Gamma^{\prime}} \subset A(\Gamma)$ its ideal. By the subscheme of $\Gamma$ residual to $\Gamma^{\prime}$ we shall mean the subscheme defined by the ideal

$$
I_{\Gamma^{\prime \prime}}=\operatorname{Ann}\left(I_{\Gamma^{\prime}} / I_{\Gamma}\right) \text {. }
$$

The problem is simply that in general this definition has neither of the properties we desire, as can be seen already in the case of $\Gamma \subset \mathbb{A}^{2}$ a "fat point", that is,

$$
I_{\Gamma}=\left(x^{2}, x y, y^{2}\right) \subset k[x, y] .
$$

If we take $\Gamma^{\prime}$ the reduced point - that is, $I_{\Gamma^{\prime}}=(x, y)$ - then by our definition the residual scheme $\Gamma^{\prime \prime}$ will again be the reduced point, which violates the degree condition. (There are also more complicated examples in which $\operatorname{deg}\left(\Gamma^{\prime}\right)+\operatorname{deg}\left(\Gamma^{\prime \prime}\right)>$ $\operatorname{deg}(\Gamma)$.$) \quad At the same time, if we take \Gamma^{\prime}$ a subscheme of degree 2 - say, for example, $I_{\Gamma^{\prime}}=\left(x, y^{2}\right)$ - then the residual scheme $\Gamma^{\prime \prime}$ to $\Gamma^{\prime}$ will be again the reduced point, violating the requirement that the residual to the residual be the original subscheme. These examples suggest the impossibility of any definition satisfying the conditions we have set. 
We are saved in the present circumstance because $\Gamma$ is not an arbitrary zerodimensional scheme; it is a complete intersection. In particular, the local rings $\mathcal{O}_{\Gamma, p}$ (which are the localizations of $A(\Gamma)$ at its maximal ideals) are Gorenstein rings, which is exactly the property we need for the definition of residuation to work.

Although Gorenstein rings are quite commonplace in commutative algebra, their usual rather abstract definition has given them the reputation in some geometric circles of being arcane and only for experts. In the present circumstance, however, we do not need the general definition: since $\Gamma$ is zero-dimensional and the rings $\mathcal{O}_{\Gamma, p}$ are correspondingly finite-dimensional vector spaces over the ground field, we can say in concrete terms what it means for the local ring $A=\mathcal{O}_{\Gamma, p}$ at a point $p \in \operatorname{supp}(\Gamma)$ to be Gorenstein. To start with the simplest definition:

Definition. Let $A$ be a local Artinian ring, $\mathfrak{m} \subset A$ its maximal ideal. We say that $A$ is Gorenstein if the annihilator of $\mathfrak{m}$ has dimension one as a vector space over $K=A / \mathfrak{m}$.

In general, for any local Artinian ring $A$ with maximal ideal $\mathfrak{m}$, the annihilator of $\mathfrak{m}$ as an $A$-module is called the socle of $A$. To see what the condition that the socle of $A$ have length 1 means, observe first of all that we know $\mathfrak{m}^{k}=0$ for $k$ large, and let $\mathfrak{m}$ be the largest integer $k$ such that $\mathfrak{m}^{k} \neq 0$. Certainly everything in $\mathfrak{m}^{m}$ annihilates $\mathfrak{m}$, so that the Gorenstein condition above at least implies that $\mathfrak{m}^{m}$ has length one, i.e.,

$$
\mathfrak{m}^{\mathrm{m}} \cong K
$$

In fact, it says more: given any nonzero $f \in A$, we claim there exists a $g \in A$ such that the product is nonzero, but $f g \in \mathfrak{m}^{\mathrm{m}}$. This is easy: if $l$ is the largest integer such that $f \cdot \mathfrak{m}^{l} \neq 0$, then any element of $f \cdot \mathfrak{m}^{l}$ annihilates $\mathfrak{m}$ and so must lie in $\mathfrak{m}^{\mathrm{m}}$.

Now suppose that $A$ contains a field. It follows from the Cohen Structure Theorem ([E, Theorem 7.7]) that $A$ contains a copy of its residue field $K$. (The reader might simply assume that $K$ is the ground field, the usual geometric situation.) The condition that the annihilator of $\mathfrak{m}$ is one-dimensional thus also implies that

Given any $K$-linear map $\rho A \rightarrow \mathfrak{m}^{\mathrm{m}}$ that restricts to

the identity on $\mathfrak{m}^{\mathrm{m}}$, the pairing

$A \times A \rightarrow A \rho \mathfrak{m}^{\mathrm{m}} \cong K$ given by multiplication

is a nondegenerate pairing on $A$ as a vector space over $K$.

In fact, it is the case conversely that the two assertions $(*)$ and $(* *)$ together are equivalent to the Gorenstein condition. To see this, observe that $(*)$ and $(* *)$ together in turn imply that there exists a $K$-linear map $A \rightarrow K$ such that the composition

$$
Q: A \times A \rightarrow A \rightarrow K
$$

is a nondegenerate pairing on the $K$-vector space $A$. On the other hand, we see that this implies the Gorenstein condition: Since the maximal ideal $\mathfrak{m} \subset A$ has codimension one as a $K$-vector space, the orthogonal complement $\mathfrak{m}^{\perp}$ of $\mathfrak{m}$ with respect to the pairing $Q$ on $A$ will have dimension one, and this certainly contains the annihilator of $\mathfrak{m}$. We thus have an alternative characterization of Gorenstein Artinian rings: 
Proposition 7. Let $A$ be an Artinian ring with residue field $K$. The ring $A$ is Gorenstein iff there exists a $K$-linear map $A \rightarrow K$ such that the composition

$$
Q: A \times A \rightarrow A \rightarrow K,
$$

where the first map is multiplication in $A$, is a nondegenerate pairing on the $K$ vector space $A$.

It may possibly be easier to visualize the Gorenstein condition in case $A$ is a graded ring. The Gorenstein condition then says that

The top graded component $A_{m}$ of $A$ has length 1, and

for every pair $k, l$ of nonnegative integers with $k+l=m$, the pairing

$$
A_{k} \times A_{l} \rightarrow A_{m}=K
$$

given by multiplication is a nondegenerate pairing of $K$-vector spaces.

Having described the Gorenstein condition for Artinian rings, it is possible to state the condition for general local rings. One bit of terminology: if $S$ is any local ring with maximal ideal $\mathfrak{m}$, we say that a sequence $\left(F_{0}, \ldots, F_{k}\right)$ of elements of $\mathfrak{m}$ is regular if for each $i=0, \ldots, k, F_{i}$ is a nonzerodivisor in $S /\left(F_{0}, \ldots, F_{i-1}\right)$; we say that $\left(F_{0}, \ldots, F_{k}\right)$ is a maximal regular sequence if it cannot be extended to a regular sequence $\left(F_{0}, \ldots, F_{k+1}\right)$, i.e., if every element of the maximal ideal of $S /\left(F_{0}, \ldots, F_{k}\right)$ is a zerodivisor. We then make the

Definition. A local ring $S$ is Gorenstein if for every maximal regular sequence $\left(F_{0}, \ldots, F_{k}\right)$ of elements of $S$ the quotient $A=S /\left(F_{0}, \ldots, F_{k}\right)$ is a Gorenstein Artinian ring, in the sense above.

For the reader's interest we mention that the ring $S$ is called Cohen-Macaulay if, in the situation above, the quotient $A$ is simply assumed Artinian; thus the Gorenstein property includes Cohen-Macaulay.

It follows from Proposition 9 below that the Gorenstein condition on the quotient $S /\left(F_{0}, \ldots, F_{k}\right)$ is independent of the maximal regular sequence $\left(F_{0}, \ldots, F_{k}\right)$, so that we could just as well replace the "every" in the preceding definition with "some". We shall put off until section 1.4 a proof that the local rings of a zero-dimensional complete intersection scheme $\Gamma$ are Gorenstein. (For a general discussion of the definitions and properties of Gorenstein rings, see Eisenbud [E, Ch. 21]; for a different discussion of the connection with Cayley-Bacharach, see Davis-Geramita-Orecchia [DGO].)

The point is that if the local rings of $\Gamma$ are Gorenstein, then our definition of the residual subscheme to a subscheme of $\Gamma$ does satisfy the two conditions we laid down: we simply observe that the ideal of the residual subscheme $\Gamma^{\prime \prime}$ to a given subscheme $\Gamma^{\prime} \subset \Gamma$ is the orthogonal complement, with respect to the pairing $Q$, of the ideal $I_{\Gamma^{\prime}}$ of $\Gamma^{\prime}$. This tells us immediately that $I_{\Gamma^{\prime}}$ and $I_{\Gamma^{\prime \prime}}$ have complementary dimensions as vector subspaces of $A$, so that $\operatorname{deg}\left(\Gamma^{\prime}\right)+\operatorname{deg}\left(\Gamma^{\prime \prime}\right)=\operatorname{deg}(\Gamma)$; and of course since the orthogonal complement of the orthogonal complement of $I_{\Gamma^{\prime}}$ will again be $I_{\Gamma^{\prime}}$, the residual subscheme of the residual subscheme of $\Gamma^{\prime}$ will again be $\Gamma^{\prime}$. With this said, we may state the next version of Cayley-Bacharach.

Theorem CB7. Let $X_{1}, \ldots, X_{n}$ be hypersurfaces in $\mathbb{P}^{n}$ of degrees $d_{1}, \ldots, d_{n}$, and suppose that the intersection $\Gamma=X_{1} \cap \cdots \cap X_{n}$ is zero-dimensional. Let $\Gamma^{\prime}$ and $\Gamma^{\prime \prime}$ be subschemes of $\Gamma$ residual to one another in $\Gamma$, and set $s=\Sigma d_{i}-n-1$. If 
$k \leq s$ is a nonnegative integer, then the dimension of the family of curves of degree $k$ containing $\Gamma^{\prime}$ (modulo those containing all of $\Gamma$ ) is equal to the failure of $\Gamma^{\prime \prime}$ to impose independent conditions of curves of complementary degree $s-k$.

A proof of this statement could be given along the lines of the preceding proof by introducing the dualizing sheaf $\omega_{X}$ of the curve (that is, one-dimensional scheme) $X=X_{1} \cap \cdots \cap X_{n-1}$ and proving a Riemann-Roch/duality type theorem relating the global sections of a sheaf $\mathcal{F}$ on $X$ to the first cohomology of the sheaf $\operatorname{Hom}\left(\mathcal{F}, \omega_{X}\right)$. But we don't have to do this: Theorem CB7 suggests yet a further (and, for now, final) generalization, from which the last statement will follow readily and which admits a relatively elementary proof! We shall now state this final version of CayleyBacharach and then indicate how it implies the last version.

Our final generalization of the Cayley-Bacharach Theorem will be expressed in terms of the homogeneous coordinate ring of $\Gamma$. We first introduce some notation. As before, we let $X_{1}, \ldots, X_{n} \subset \mathbb{P}^{n}$ be hypersurfaces intersecting in a zerodimensional scheme $\Gamma$. We let $S=K\left[Z_{0}, \ldots, Z_{n}\right]$ be the homogeneous coordinate ring of $\mathbb{P}^{n}$ and let $F_{i}(Z)$ be the homogeneous polynomial of degree $d_{i}$ defining the hypersurface $X_{i}$. We write $I(\Gamma) \subset S$ for the ideal of homogeneous polynomials vanishing on $\Gamma$ and set $R=S / I(\Gamma)$, the homogeneous coordinate ring of $\Gamma$. In keeping with our algebraic point of view, we will now write $h_{R}(k)$ for what we used to call $h_{\Gamma}(k)$, the number of conditions imposed by $\Gamma$ on forms of degree $k$; it is just the vector space dimension of $R_{k}$, the $k$ th graded component of $R$.

We have had occasion to use Max Noether's $A F+B G$ theorem several times already, and we shall need a more powerful version of it in the sequel. This is the Lasker's Unmixedness Theorem, which extends Noether's Theorem to an assertion about homogeneous sequences of parameters. We say that a sequence of homogeneous forms $F_{1}, \ldots, F_{s}$ is a sequence of parameters if the algebraic set defined by the vanishing of $F_{1}, \ldots, F_{s}$ (either in $\mathbb{A}^{n+1}$ or in $\mathbb{P}^{n}$, it comes to the same thing) has codimension $s$, the number of forms.

Theorem 8 (Lasker). If $F_{1}, \ldots, F_{s} \in S$ is a sequence of parameters and $\Gamma \neq \varnothing$ is the scheme in $\mathbb{P}^{n}$ that is the intersection of the hypersurfaces defined by the $F_{i}$, then every polynomial vanishing on $\Gamma$ is a linear combination of the $F_{i}$; equivalently (in case the ground field is infinite) there exists a linear form $L$ that is a nonzerodivisor modulo $\left(F_{1}, \ldots, F_{s}\right)$.

Noether's Theorem is just the case $n=2$. (The development of Noether's Theorem does not end here: it was extended by Macaulay [M, sections 48-53] to systems of parameters in other rings and became the basis of the theory of CohenMacaulay rings, which may be defined as local rings for which the Unmixedness Theorem is true; see Nagata $[\mathrm{Na}]$.

Next, we do something that may strike the geometrically oriented reader as a bit odd: we let $H \subset \mathbb{P}^{n}$ be a general hyperplane (specifically, one not meeting the support of $\Gamma), L$ the linear form defining $H$, and introduce the quotient ring

$$
A=R /(L)=S /\left(L, F_{1}, \ldots, F_{n}\right) .
$$

Now, if we think of $R$ simply as the homogeneous coordinate ring of the projective scheme $\Gamma$, this makes no apparent sense: the ideal $\left(L, F_{1}, \ldots, F_{n}\right)$ defines the empty set. It makes much more sense if we think of $R$ as the affine coordinate ring of the cone $\widetilde{\Gamma}$ over $\Gamma$ in affine space $\mathbb{A}^{n+1}$ (Figure 4): then $A$ is the coordinate ring of the hyperplane section of $\widetilde{\Gamma}$ corresponding to a hyperplane meeting $\widetilde{\Gamma}$ at the origin, and 


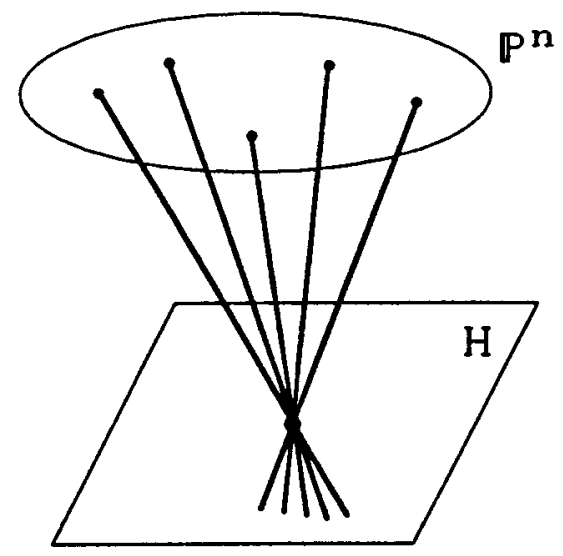

FigURE 4

it is not unreasonable to hope that the scheme structure of this intersection reflects the global geometry of $\Gamma$.

To see how this works, observe that $R$ and $A$ are graded rings and that $L$ is a nonzerodivisor in $R$. It follows that the Hilbert function of $A$ (which is again a graded ring) will be the first difference function of the Hilbert function of $R$ :

$$
h_{A}(m)=h_{R}(m)-h_{R}(m-1) .
$$

Moreover, the same is true for any quotient $R^{\prime}=R / I^{\prime}$ and corresponding $A^{\prime}=$ $R /\left(I^{\prime}, L\right)$, as long as $L$ is a nonzerodivisor modulo $I^{\prime}$. Thus for any subscheme $\Gamma^{\prime} \subset \Gamma$ we let $\overline{I^{\prime}}$ be the image in $A=R /(L)$ of the ideal $I^{\prime}$ of $\Gamma$ and write

$$
\begin{aligned}
h^{0}\left(\mathbb{P}^{n}, I_{\Gamma^{\prime}}(k)\right)-h^{0}\left(\mathbb{P}^{n}, I_{\Gamma}(k)\right) & \\
= & h_{R}(k)-h_{R^{\prime}}(k) \\
= & \sum_{j=0}^{k}\left(h_{A}(j)-h_{A^{\prime}}(j)\right) \\
= & \sum_{j=0}^{k} \operatorname{dim}_{K}\left(\bar{I}^{\prime}{ }_{j}\right) .
\end{aligned}
$$

Now let $i(l)$ denote the failure of the subscheme $\Gamma^{\prime}$ to impose independent conditions on hypersurfaces of a given degree $l$. It is likewise the case that we can measure $i(l)$ in terms of the ring $R^{\prime}$ : we have

$$
i(l)=\operatorname{deg}\left(\Gamma^{\prime}\right)-h_{R^{\prime}}(l)
$$

so that the successive differences

$$
i(l)-i(l+1)=h_{R^{\prime}}(l+1)-h_{R^{\prime}}(l) .
$$

Thus

$$
i(l)=\sum_{j=l+1}^{\infty} h_{R^{\prime} /(L)}(l) .
$$

Of course, the sum need only extend over the finite range of $j \geq l+1$ for which ${\overline{I^{\prime}}}_{j} \varsubsetneqq R_{j}$, that is, $h_{R^{\prime} /(L)}(l) \neq 0$. 
The crucial result, which we prove in the next section, is that the $\operatorname{ring} A$ is itself Gorenstein, with socle in degree $m$. This means that we get a nondegenerate pairing

$$
Q: A \times A \rightarrow A \rightarrow A_{m} \cong K
$$

Let $\Gamma^{\prime \prime} \subset \Gamma$ be the subscheme residual to $\Gamma$, and let $I^{\prime \prime} \subset R$ be its idea; let $\overline{I^{\prime \prime}}$ in $A$. We observe that the ideal $\overline{I^{\prime \prime}} \subset R$ is just the annihilator of $\overline{I^{\prime}}$ with respect to the pairing $Q$ : clearly they annihilate each other, and the sum of their codimensions (as $K$-vector spaces) in $A$ is equal to the dimension $d$ of $R$. In particular, the dimension of the $j$ th graded piece of $\overline{I^{\prime}}$ is the codimension of the $(m-j)$ th graded piece of $\overline{I^{\prime \prime}}$.

Summing this equality over $j=0,1, \ldots, l$ yields the statement: the number of hypersurfaces of degree $l$ containing $\Gamma^{\prime}$ (modulo those containing $\Gamma$ ) is equal to the failure of $\Gamma^{\prime \prime}$ to impose independent conditions on hypersurfaces of degree $m-1-l$. This, of course, is exactly the statement of our last version of Cayley-Bacharach! Cayley-Bacharach thus turns out to be simply the statement that the $\operatorname{ring} A$ is Gorenstein, in other words, the special case $d_{0}=1$ of the

Theorem CB8. If $F_{0}, \ldots, F_{n} \in K\left[Z_{0}, \ldots, Z_{n}\right]$ are homogeneous polynomials of degrees $d_{0}, \ldots, d_{n}$ forming a sequence of parameters (i.e., having no common zeros in $\left.\mathbb{P}^{n}\right)$, then the ring

$$
A=K\left[Z_{0}, \ldots, Z_{n}\right] /\left(F_{0}, \ldots, F_{n}\right)
$$

is Gorenstein, with socle in degree $\Sigma d_{i}-n-1$.

Note that in terms of the definition of the Gorenstein condition this statement is equivalent (modulo specifying the degree of the socle, which is elementary) to the shorter

Theorem CB9. The polynomial ring $K\left[Z_{0}, \ldots, Z_{n}\right]$ is Gorenstein.

This is our final version of the Cayley-Bacharach Theorem. Whether it does in fact convey a deeper understanding of the phenomena expressed in the original theorems of Pappus, Pascal, and Chasles, or represents merely the addition of a layer of technical machinery, we leave to the reader. Perhaps the best criterion is the simplicity of the proof, which appears in the following section.

1.4. Proof of the final Cayley-Bacharach Theorem. In this section we shall, as advertised, give a proof "from scratch" of the final version of the CayleyBacharach Theorem. We also owe the reader the verifications of two statements made along the way: that the local rings of a zero-dimensional complete intersection subscheme $\Gamma \subset \mathbb{P}^{n}$ are Gorenstein (the reader will recognize this as a weaker version of the final Cayley-Bacharach Theorem) and that the ideal $\left(F_{1}, \ldots, F_{k}\right) \subset$ $K\left[Z_{1}, \ldots, Z_{n}\right]$ generated by a sequence of parameters is saturated.

As for the proofs, these involve properties of the Ext functor. They are nonetheless elementary in spirit: in fact, all we shall use is the basic definition of the Ext functors as right-derived functors of the functor Hom. In particular, we do not actually calculate any Ext groups (other than Hom) or use any of their geometric or algebraic properties. Rather, as is often the case with homological functors, we simply use the exact sequences associated to them to transport information from one setting to another, in this case, from one quotient of the polynomial ring to another. 
To begin with, one piece of terminology. Let $S$ be any local ring with maximum ideal $\mathfrak{m}$ and residue field $K$, and $M$ any finitely generated graded $S$-module. We say that a sequence $\left(F_{0}, \ldots, F_{k}\right)$ of elements of $\mathfrak{m}$ is an $M$-regular sequence (or just $M$-sequence) if for each $i=0, \ldots, k, F_{i}$ is a non-zero-divisor for the module $M /\left(F_{0}, \ldots, F_{i-1}\right) M$. Of course, we say that $\left(F_{0}, \ldots, F_{k}\right)$ is a maximal $M$-sequence if it cannot be extended to an $M$-sequence $\left(F_{0}, \ldots, F_{k+1}\right)$ that is, if every element of $\mathfrak{m}$ is a zerodivisor for $M /\left(F_{0}, \ldots, F_{k}\right) M$.

All the needed results will follow directly from

Proposition 9. With the above notation, if $\left(F_{0}, \ldots, F_{k}\right)$ is a maximal $M$-sequence, then

$$
\begin{aligned}
\operatorname{Ext}^{i}(K, M) & =0 \quad \text { for all } i \leq k, \text { and } \\
\operatorname{Ext}^{k+1}(K, M) & =\operatorname{Hom}\left(K, M /\left(F_{0}, \ldots, F_{k}\right) M\right) \neq 0 .
\end{aligned}
$$

Before we prove this, let's see how it implies all the assertions made so far about the algebra of the rings $R$ and $A$ associated to a complete intersection in $\mathbb{P}^{n}$.

To begin with, the most immediate implication is simply that all maximal $M$ sequences contain the same number $k$ of elements, $k$ being characterized as the smallest integer such that $\operatorname{Ext}^{k}(K, M) \neq 0$. In particular, in case $M=S$ we see that all maximal regular sequences in the ring $S$ have the same number of elements; this number is called the depth of $S$. Now, in case $S=K\left[Z_{0}, \ldots, Z_{n}\right]$ is the polynomial ring, $\left(Z_{0}, \ldots, Z_{n}\right)$ is visibly a maximal regular $S$-sequence, and it follows then that all maximal regular sequences in $K\left[Z_{0}, \ldots, Z_{n}\right]$ have $n+1$ elements. This, finally, means that any regular sequence $\left(F_{1}, \ldots, F_{n}\right)$ is not maximal, i.e., that the ring $K\left[Z_{0}, \ldots, Z_{n}\right] /\left(F_{1}, \ldots, F_{n}\right)$ contains nonzerodivisors of positive degree, i.e., that the ideal $\left(F_{1}, \ldots, F_{n}\right) \subset S$ is saturated.

Next, observe that the socle of a local Artinian ring $A$ with residue field $K$ is the module $\operatorname{Hom}(K, A)$; thus in particular $A$ will be Gorenstein if and only if $\operatorname{Hom}(K, A) \cong K$. Now, applying the Proposition with $M=S$, we see that if $S$ is any local ring with residue field $K$ and $\left(F_{0}, \ldots, F_{k}\right)$ is a maximal regular sequence in $S$, then

$$
\operatorname{Hom}_{A}(K, A)=\operatorname{Hom}_{S}(K, A)=\operatorname{Ext}^{k+1}(K, S)
$$

is independent of the particular maximal regular sequence! Thus one quotient $S /\left(F_{0}, \ldots, F_{k}\right)$ will be Gorenstein only if any such quotient is. We have thus verified the remark made immediately following the definition of Gorenstein rings. In particular, since the quotient $K\left[Z_{0}, \ldots, Z_{n}\right] /\left(Z_{0}, \ldots, Z_{n}\right) \cong K$ is trivially Gorenstein, it follows that $K\left[Z_{0}, \ldots, Z_{n}\right] /\left(F_{0}, \ldots, F_{n}\right)$ is Gorenstein for any maximal regular sequence $\left(F_{0}, \ldots, F_{n}\right)$, thus completing the proof of Theorem CB9.

Proof of Proposition 9. The argument here will be by induction on $k$, the inductive step being easy and the hard part being the first case $k=0$. For the inductive step, we just observe that since $F_{0}$ is a nonzerodivisor for the module $M$, we have an exact sequence of $S$-modules

$$
0 \rightarrow M \rightarrow M \rightarrow M / F_{0} M \rightarrow 0
$$

where the first map is multiplication by $F_{0}$. Moreover, since $F_{0} \in \mathfrak{m}$ annihilates the module $K=S / \mathfrak{m}$, the induced map on $\operatorname{Ext}^{*}(K, M)$ is zero. Thus we have for each $l$ a three-term exact sequence

$$
0 \rightarrow \operatorname{Ext}^{l}(K, M) \rightarrow \operatorname{Ext}^{l}\left(K, M / F_{0} M\right) \rightarrow \operatorname{Ext}^{l+1}(K, M) \rightarrow 0
$$


which establishes the inductive step: assuming the result for the module $M / F_{0} M$, with its maximal regular sequence $F_{1}, \ldots, F_{k}$, we deduce first that $\operatorname{Ext}^{i}(K, M)=0$ for $i \leq k$ and then that

$$
\begin{aligned}
\operatorname{Ext}^{k+1}(K, M) & \cong \operatorname{Ext}^{k}\left(K, M / F_{0} M\right) \\
& \cong \operatorname{Hom}\left(K, M /\left(F_{0}, \ldots, F_{k}\right) M\right) \neq 0 .
\end{aligned}
$$

The hard part, as we indicated, is the initial step. We know that if $k \geq 0$ i.e., $\mathfrak{m}$ does contain a nonzerodivisor for $M$-then $\operatorname{Hom}(K, M)=0$; we have to establish that conversely if $k=-1$ - that is, if every element of $\mathfrak{m}$ is a zerodivisor for $M$-then $\operatorname{Hom}(K, M) \neq 0$. This amounts to the statement of

Proposition 10. If every element $F \in \mathfrak{m}$ kills some element of $M$, then there exists an element $\alpha \in M$ killed by all of $\mathfrak{m}$.

Proof. For each element $\alpha \in M$, let $I_{\alpha} \subset S$ be the ideal $\operatorname{Ann}(\alpha)$ of elements of $S$ that kill $\alpha$; our hypothesis is that $\mathfrak{m}$ is the union of the ideals $I_{\alpha}$. Of course, we need only look at the maximal elements of the set of ideals $\left\{I_{\alpha}\right\}$, and these are prime ideals: if $I_{\alpha}$ is maximal, and $F G \in I_{\alpha}$ and $G \notin I_{\alpha}$ (i.e., $G \alpha \neq 0$ ), then $I_{G \alpha} \supset I_{\alpha}$, and hence $I_{G \alpha}=I_{\alpha}$ and $F \in I_{G \alpha}=I_{\alpha}$. In general, maximal elements of the set of annihilators of elements of a module $M$ are called associated primes of $M$.

We claim next that since $S$ is Noetherian and $M$ is finitely generated, there can be only finitely many such primes: if $I_{\alpha}$ is any such prime, we have a sequence

$$
0 \rightarrow A \cdot \alpha \cong A / I_{\alpha} \rightarrow M \rightarrow M^{\prime} \rightarrow 0 .
$$

Note that since $A / I_{\alpha}$ is an integral domain, if $\beta \in A \cdot \alpha$ is any nonzero element, then $I_{\beta}=I_{\alpha}$. Thus, if $I_{\gamma}$ is any other such prime, $A \cdot \alpha \cap A \cdot \gamma=(0)$, so that $\gamma$ is an associated prime of $M^{\prime}$. If $M^{\prime}$ has only finitely many associated primes, then it follows that $M$ does, and the result follows by Noetherian induction.

We have thus expressed the maximal ideal $\mathfrak{m}$ of $S$ as a finite union of prime ideals $I_{\alpha}$. It follows that $\mathfrak{m}=I_{\alpha}$ for some $\alpha$, i.e., there exists an element of $M$ killed by all of $\mathfrak{m}$. If $S$ contains an infinite field, as in the original geometric case, this is trivial, as a vector space over an infinite field cannot be the union of finitely many proper subspaces; otherwise, it is what is called in commutative algebra the "prime avoidance lemma". See, for example, Eisenbud [E, section 3.2].

\section{PART II: The FUture?}

2.1. Cayley-Bacharach Conjectures. The statement of Theorem CB9 represents our best understanding (to date) of the phenomena observed in the various formulations of Cayley-Bacharach preceding it; having expressed the result as a relationship between the Hilbert functions of residual subsets of an arithmetically Gorenstein subscheme of $\mathbb{P}^{n}$, we do not see at present how to extend it to any larger class of objects. What we would like to propose instead is an extension of the theorem in a different direction, toward a collection of inequalities on the Hilbert function of a subscheme of a complete intersection.

To see this most clearly, we restrict our attention to the case of a complete intersection $\Gamma \subset \mathbb{P}^{n}$ of $n$ quadrics; we shall give the general version at the end. Cayley-Bacharach says that any homogeneous polynomial of degree $2 n-(n+1)=$ $n-1$ on $\mathbb{P}^{n}$ containing a subscheme $\Gamma_{0}$ of colength 1 of $\Gamma$ (that is, of degree $2^{n}-1$ ) must contain all of $\Gamma$; we consider now what can be said about hypersurfaces of other degrees. Of course, Cayley-Bacharach also says that $\Gamma$ does impose independent 
conditions on polynomials of degree $n$, so there will exist polynomials of degree $n$ vanishing on a subscheme of $\Gamma$ of colength 1 but not on $\Gamma$. On the other hand, by Bézout, a hyperplane in $\mathbb{P}^{n}$ can contain a subscheme of $\Gamma$ of degree at most $2^{n-1}$.

To summarize what we know, define for any given $n$ a function $d$ by saying that $d(k)$ is the largest degree of a subscheme $\Gamma_{0}$ of a complete intersection $\Gamma$ such that there is a polynomial $F(Z)$ of degree $k$ vanishing on $\Gamma_{0}$ but not on $\Gamma$. Throwing in the trivial case of degree 0 , we have then the four values of $d$ :

$$
\begin{aligned}
& \text { If } k=n, \text { then } d(k)=2^{n}-1 \\
& \text { If } k=n-1, \text { then } d(k)=2^{n}-2 \\
& \cdot \\
& \text { If } k=1, \\
& \text { If } k=0 \text {, then } d(k)=2^{n}-2^{n-1}
\end{aligned}
$$

and the pattern is clear. We may make, accordingly, the

Conjecture CB10. Let $\Gamma$ be a complete intersection of $n$ quadrics in $\mathbb{P}^{n}$. If $X \subset$ $\mathbb{P}^{n}$ is any hypersurface of degree $k$ containing a subscheme $\Gamma_{0}$ of degree strictly greater than $2^{n}-2^{n-k}$, then $X$ contains $\Gamma$.

Note that this conjecture is sharp, if true: for any $k<n$, we can find a complete intersection $\Gamma \subset \mathbb{P}^{n}$ containing a complete intersection $\Omega \subset \mathbb{P}^{n-k} \subset \mathbb{P}^{n}$; by CayleyBacharach the residual scheme $\Gamma_{0}$ to $\Omega$ in $\Gamma$ will then lie on a hypersurface of degree $k$ not containing $\Gamma$. In fact, these are the only known examples of equality; we may make the further conjecture that if $X$ is a hypersurface of degree $k$ with $\operatorname{deg}(X \cap \Omega)=2^{n}-2^{n-k}$, the scheme residual to $X \cap \Omega$ in $\Omega$ is a complete intersection of quadrics in a subspace $\mathbb{P}^{n-k}$.

It should be said that taking $\Gamma$ a complete intersection of quadrics specifically is not essential; there is a form of the conjecture applicable to arbitrary zerodimensional complete intersection subschemes of $\mathbb{P}^{n}$. As might be expected, though, this form is substantially more complicated, and the pattern of known results less apparent [EGH2].

It should also be said that we did not arrive at this conjecture by trying to extrapolate (or interpolate) from the classical statement of Cayley-Bacharach, as we do above; rather, as described in Eisenbud-Green-Harris [EGH1], we were led to this by extending a series of results and conjectures in Castelnuovo theory from Eisenbud-Harris [EH1] and made the connection to the Cayley-Bacharach Theorem after the fact.

In the remainder of this paper we shall be concerned with proving cases of the Cayley-Bacharach conjecture. In order to do this, we start by introducing a related conjecture.

Conjecture CB11. Let $\Gamma$ be any subscheme of a zero-dimensional complete intersection of quadrics in any projective space $\mathbb{P}^{r}$. If $\Gamma$ fails to impose independent conditions on hypersurfaces of degree $m$, then

$$
\operatorname{deg}(\Gamma) \geq 2^{m+1} .
$$


Here again this statement is sharp, if true: a complete intersection in $\mathbb{P}^{m+1}$ provides examples of equality for each $m$. As in the case of Conjecture CB10, moreover, these are the only examples, and we may conjecture further that equality holds in Conjecture CB11 if and only if $\Gamma$ is itself a complete intersection of quadrics in $\mathbb{P}^{m+1}$.

Theorem 11. The following are equivalent:

a. Conjecture CB10 for all $k$ and $n$;

b. Conjecture CB11 for all $m$.

Proof. We first prove that Conjecture CB11 for a given value of $m$ implies Conjecture CB10 in case $k=n-m-1$. We do this simply by applying the seventh version of the Cayley-Bacharach Theorem. To begin with, assume Conjecture CB11 for a given value of $m$, and let $\Omega \subset \mathbb{P}^{n}$ be a complete intersection of quadrics. Let $X$ be any hypersurface of degree $k=n-m-1$ not containing $\Omega$, and let $\Gamma$ be the subscheme of $\Omega$ residual to the intersection $\Omega \cap X$. By Cayley-Bacharach $\Gamma$ must fail to impose independent conditions on hypersurfaces of degree $m=n-1-k$. By assumption $\operatorname{deg}(\Gamma) \geq 2^{n-k}$ and correspondingly $\operatorname{deg}(X \cap \Omega) \leq 2^{n}-2^{n-k}$.

Now assume Conjecture CB10 for all $n$ and $k$. Let $\Gamma$ be any subscheme of a complete intersection of quadrics, and suppose that $\Gamma$ fails to impose independent conditions on hypersurfaces of degree $m$. Assuming that $\Gamma$ spans a projective space $\mathbb{P}^{n}$, take $\Omega$ a complete intersection of quadrics in $\mathbb{P}^{n}$ containing $\Gamma$, and let $\Gamma^{\prime} \subset \Omega$ be the subscheme of $\Omega$ residual to $\Gamma$. By Cayley-Bacharach, $\Gamma^{\prime}$ lies on a hypersurface of degree $n-1-m$ not containing $\Omega$; it follows that $\operatorname{deg}\left(\Gamma^{\prime}\right) \leq 2^{n}-2^{m+1}$ and hence that $\operatorname{deg}(\Gamma) \geq 2^{m+1}$.

Note as well that, by the proof of Theorem 11, Conjecture CB11 for all $m \leq m_{0}$ implies Conjecture CB11 for all $k$ and $n$ such that

$$
n-k \leq m_{0}+1
$$

and since Conjecture CB10 is immediate in case $k=n$ or $n-1$, it follows that Conjecture CB11 for all $m \leq m_{0}$ implies Conjecture CB10 for all $k$ and $n$ with $n \leq m_{0}+3$.

Finally, we formulate a general version of our Conjecture CB11 that does not require quadrics.

Conjecture CB12. Let $\Gamma$ be any subscheme of a zero-dimensional complete intersection of hypersurfaces of degrees $d_{1} \leq \cdots \leq d_{n}$ in a projective space $\mathbb{P}^{n}$. If $\Gamma$ fails to impose independent conditions on hypersurfaces of degree $m$, then

$$
\operatorname{deg}(\Gamma) \geq e \cdot d_{s} \cdot d_{s+1} \cdot \cdots \cdot d_{n}
$$

where $e$ and $s$ are defined by the relations

$$
\sum_{i=s}^{n}\left(d_{i}-1\right) \leq m+1<\sum_{i=s-1}^{n}\left(d_{i}-1\right)
$$

and

$$
e=m+1-\sum_{i=s}^{n}\left(d_{i}-1\right) .
$$

Note that this is the analogue of our second Cayley-Bacharach conjecture. It may in turn be translated, by an argument generalizing Theorem 11, into a statement analogous to Conjecture CB10. 
2.2. A proof of Conjecture CB10 in case $r \leq 7$. We shall here prove Conjecture CB11 in case $m \leq 4$ and correspondingly Conjecture CB10 when $r \leq 7$.

We start with an arbitrary zero-dimensional subscheme $\Gamma \subset \mathbb{P}^{r}$. As before, we introduce the coordinate ring $S=S(\Gamma)$ of $\Gamma$, and we let $R=S /(L)$ be the quotient of $S$ by a general linear form $L$. $R$ is then a graded local Artinian ring over the field $K$, generated by its first graded piece $R_{1}$, whose dimension as a vector space over $K$ we shall call $n$. By the ideal of $R$ we shall mean the ideal of relations among the generators of $R$, that is, the kernel $I$ of the surjection from $\operatorname{Sym}^{*} R_{1}$ onto $R$. We may then translate our geometric hypotheses on the scheme $\Gamma \subset \mathbb{P}^{r}$ into statements about the ring $R$ as follows:

The degree of $\Gamma$ is the length of $R$ (i.e., the dimension of $R$ as a vector space over $K)$.

The dimension $n$ of $R_{1}$ as a vector space over $K$ is the dimension of the span of $\Gamma$ in $\mathbb{P}^{r}$.

The statement that $\Gamma$ fails to impose independent conditions on hypersurfaces of degree $m$ is equivalent to the statement that the $(m+1)$ st graded piece $R_{m+1}$ of $R$ is nonzero.

The statement that $\Gamma$ is a subscheme of a complete intersection of quadratics amounts to the assertion that the ideal of $R$ contains a regular sequence of length $n$ in degree 2 .

With these conventions, the result from which our conjecture will follow in case $r \leq 7$ is the

Proposition 12. Let $R$ be a graded local Artinian ring over the field $K$, generated by its first graded piece $R_{1}$; suppose that the ideal of $R$ contains a regular sequence of length $n=\operatorname{dim}_{K}\left(R_{1}\right)$ in degree 2 . For $k \leq 5$, if the $k$ th graded piece $R_{k}$ of $R$ is nonzero, then the length of $R$ is at least $2^{k}$.

Proof. Note first that we may as well assume $R$ to be Gorenstein: if the socle of $R$ has vector space dimension strictly bigger than 1 , we could simply replace $R$ by the quotient of $R$ by any subspace of the socle not containing $R_{k}$ to obtain a ring of smaller length.

Now, we break up the argument into two cases: If we assume that the ideal $I$ of $R$ contains a reducible quadric, then as we shall see we may reduce the problem to one of rings $R^{\prime}$ with socles in lower degree and proceed by induction. If, on the other hand, $I$ contains no such element, we obtain lower bounds on the Hilbert function of $R$ that suffice, at least in case $k \leq 5$, to establish the desired inequality on the length of $R$.

Assume first that the ideal $I$ of $R$ contains a reducible quadric, that is, that we have $x y=0$ for some pair of nonzero elements $x, y \in R_{1}$ (we do not assume $x$ and $y$ are linearly independent). From the exact sequence of $R$-modules

$$
0 \rightarrow(x) \rightarrow R \rightarrow R /(x) \rightarrow 0
$$

we have the obvious equality

$$
l(R)=l((x))+l(R /(x))
$$

where $l$ denotes length. On the other hand, the ideals $(x)$ and $(y)$ in $R$ have as well the structure of quotient rings of $R$ : for example, $(y)$ is just the image of the map $m_{y}: R \rightarrow R$ of $R$-modules given by multiplication by $y$ and so is isomorphic to the quotient ring $R / \operatorname{Ann}(y)$. Moreover, since $x y=0$ in $R, x$ is in the kernel of $m_{y}$, so 
that in fact the ring $(y)$ is a quotient of the ring $R(x)$. In particular, the length of $(y)$ is less than or equal to that of $R /(x)$, so that we have

$$
l(R) \geq l((x))+l((y)) .
$$

Now, the rings $(x)$ and $(y)$, being quotients of the ring $R$, are likewise generated by their first graded pieces; similarly, their ideals contain regular sequences of maximal length in degree 2. Moreover, they are again Gorenstein. To see this, observe that every ideal of $R$ contains the socle $R_{k}$ of $R$, so that for example $R_{k} \subset(x)$ and is indeed contained in the socle of the ring $(x)$. At the same time, since $(x)$ is a submodule of the $R$-module $R$, any element of the socle of $(x)$ is killed by any element of $R_{1}$ and so is an element of the socle of $R$. Indeed, we see that $(x)$ and $(y)$ have socles in degree exactly $k-1$, and by induction we may conclude that each has length at least $2^{k+1}$. Thus

$$
l(R) \geq l((x))+l((y)) \geq 2^{k}
$$

and we are done in this case.

Suppose now that the ideal of $R$ contains no reducible element in degree 2 . If as before we let $n$ denote the dimension of the vector space $R_{1}$, then in the projective space $\mathbb{P}\left(\operatorname{Sym}^{2}\left(R_{1}\right)\right)$ the locus $\Sigma$ of reducible elements has dimension $2 n-2$. Inasmuch as the kernel $I_{2}$ of the map

$$
\operatorname{Sym}^{2}\left(R_{1}\right) \rightarrow R_{2}
$$

does not meet $\Sigma$, we may immediately conclude that the codimension of $I_{2}$ in $\operatorname{Sym}^{2}\left(R_{1}\right)$ is at least $2 n-1$. We have thus

$$
\operatorname{dim}_{K}\left(R_{2}\right) \geq 2 n-1 \text {. }
$$

Now, under the hypothesis that the ideal of $R$ contains a regular sequence $f_{1}, \ldots, f_{n}$ of maximal length in degree 2 , it is a quotient of the ring

$$
\operatorname{Sym}^{*}\left(R_{1}\right) /\left(f_{1}, \ldots, f_{n}\right),
$$

which has length $2^{n}$ and socle in degree $n$. Thus we must have $n \geq k$, and the result we are after is immediate if $n=k$, so we may as well assume that $n \geq k+1$. The Hilbert function $h_{R}$ thus satisfies

$$
\begin{aligned}
& h_{R}(0)=1 \\
& h_{R}(1)=n \geq k+1 \\
& h_{R}(2) \geq 2 n-1
\end{aligned}
$$

from which we conclude immediately that $k \geq 4$ : if $k=1$, we would have $h_{R}(2)=0$; if $k=2$, we would have $h_{R}(2)=1$; and if $k=3$, we would have $h_{R}(2)=h_{R}(1)$. Moreover, in case $k=4$ or 5 the entire Hilbert function is determined by the first three values above, and we can just add up the inequalities we have to obtain the result. For example, in case $k=4$ we have $h_{R}(3)=h_{R}(1)=n \geq k+1$ and 
$h_{R}(4)=1$. Adding it all up, we see that the length of $R$ must be

$$
\begin{aligned}
l(R) & =\sum h_{R}(k) \\
& \geq 1+n+2 n-1+n+1 \\
& =4 n+1 \\
& \geq 4 k+5 \\
& =21 \\
& >2^{k},
\end{aligned}
$$

so we are done. Similarly, in case $k=5$ we have $h_{R}(3)=h_{R}(2) \geq 2 n-1, h_{R}(4)=$ $h_{R}(1)=n$, and $h_{R}(5)=1$, so that

$$
\begin{aligned}
l(R) & =\sum h_{R}(k) \\
& \geq 1+n+2 n-1+2 n-1+n+1 \\
& =6 n \\
& \geq 6 k+6 \\
& =36 \\
& >2^{k} . \quad \square
\end{aligned}
$$

\section{REFERENCES}

[Ba] I. Bacharach, Uber den Cayley'schen Schnittpunktsatz, Math. Ann. 26 (1886), 275-299.

$[\mathrm{BM}]$ C. B. Boyer and U. Merzbach, A history of mathematics, second ed., Wiley, New York, 1991. MR 92a:01003

[BN] A. Brill and M. Noether, Uber die algebraischen Functionen und ihre Anwendung in der Geometrie, Math. Ann. 7 (1874), 269-310.

[Co] H. S. M. Coxeter, Projective geometry, second ed., Univ. of Toronto Press, Toronto, 1974. MR 49:11377

[Ca] A. Cayley, On the intersection of curves, Cambridge Math. J. 3 (1843), 211-213; Collected math papers I, vols. 25-27, Cambridge Univ. Press, Cambridge, 1889.

[Ch] M. Chasles, Traité des sections coniques, Gauthier-Villars, Paris, 1885.

[DGO] E. Davis, A. V. Geramita, and F. Orecchia, Gorenstein algebras and the CayleyBacharach Theorem, Proc. Amer. Math. Soc. 93 (1985), 593-597. MR 86k:14034

[E] D. Eisenbud, Commutative algebra. With a view toward algebraic geometry, SpringerVerlag, New York, 1994. MR 1:322 960

[EH1] D. Eisenbud and J. Harris, Castelnuovo theory, Curves in Projective Space, Univ. of Montreal Press, Montreal, 1982. MR 84g:14024

[EH2] - Schemes: The language of modern algebraic geometry, Wadsworth, Belmont, CA, 1992. (To be republished in revised form by Springer-Verlag with the title Why schemes?) MR 83k:14001

[EGH1] D. Eisenbud, M. Green, and J. Harris, Some conjectures extending Castelnuovo Theory, Astérisque 218 (1993), 187-202. MR 95a:14057

[EGH2] _ Hilbert functions and complete intersections (in preparation).

[EP] P. Ellia and C. Peskine, Groupes de points de $P^{2}$ : Caractére et position uniforme, Algebraic Geometry (L'Aquila 1988), A. Sommese, A. Biancofiore, E. Livorni, Springer Lect. Notes in Math. 1417 (1990), 111-116. MR 91f:14025

[GKR] A. V. Geramita, M. Kreuzer, and L. Robbiano, Cayley-Bacharach schemes and their canonical modules, Trans. Amer. Math. Soc. 339 (1993), 163-189. MR 93k:14065

[H] R. Hartshorne, Algebraic geometry, Springer-Verlag, New York, 1977. MR 57:3116

[K] M. Kline, Mathematical thought from ancient to modern times, Oxford Univ. Press, Oxford, 1972. MR 57:12010

[M] F. S. Macaulay, Algebraic theory of modular systems, Cambridge Tracts in Math., vol. 19, Cambridge Univ. Press, Cambridge, 1916. 
[Na] M. Nagata, The theory of multiplicity in general local rings, Proc. Internat. Sympos. (Tokyo-Nikko, 1955), Sci. Council of Japan, Tokyo, 1956, pp. 191-226. MR 18:637b

[No] M. Noether, Uber ein Satz aus der Theorie der algebraischen Funktionen, Math. Ann. 6 (1873), 351-359.

[S] D. Struik, A source book in mathematics, 1200-1800, Harvard Univ. Press, Cambridge, MA, 1969. MR 39:11

Department of Mathematics, Brandeis University, Waltham, Massachusetts 022549110

E-mail address: eisenbud@math.brandeis.edu

Department of Mathematics, University of California, los Angeles, Los Angeles, CALIFORNia 90095-1555

E-mail address: mlg@math.ucla.edu

Department of Mathematics, Harvard University, Cambridge, Massachusetts 021382901

E-mail address: harris@abel.math.harvard.edu 